\title{
Front Matter: Volume 9905
}

, "Front Matter: Volume 9905," Proc. SPIE 9905, Space Telescopes and Instrumentation 2016: Ultraviolet to Gamma Ray, 990501 (15 September 2016); doi: 10.1117/12.2240597

Event: SPIE Astronomical Telescopes + Instrumentation, 2016, Edinburgh, United Kingdom 


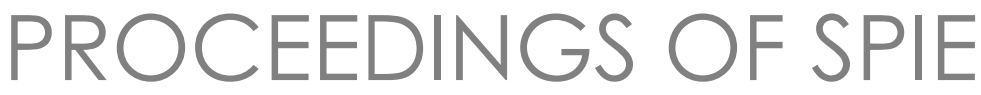

\title{
Space Telescopes and Instrumentation 2016: Ultraviolet to Gamma Ray
}

\author{
Jan-Willem A. den Herder \\ Tadayuki Takahashi \\ Marshall Bautz \\ Editors
}

26 June - 1 July 2016

Edinburgh, United Kingdom

Sponsored by

SPIE

Cooperating Organizations

American Astronomical Society (United States) - Australian Astronomical Observatory (Australia) • Association of Universities for Research in Astronomy (AURA) Canadian Astronomical Society (CASCA) (Canada) - Canadian Space Agency (Canada) European Astronomical Society (Switzerland) • European Southern Observatory (Germany) National Radio Astronomy Observatory • Royal Astronomical Society (United Kingdom) Science \& Technology Facilities Council (United Kingdom)

Published by

SPIE

Volume 9905

Part One of Three Parts

Proceedings of SPIE 0277-786X, V. 9905

SPIE is an international society advancing an interdisciplinary approach to the science and application of light.

Space Telescopes and Instrumentation 2016: Ultraviolet to Gamma Ray, edited by

Jan-Willem A. den Herder, Tadayuki Takahashi, Marshall Bautz, Proc. of SPIE Vol. 9905, 990501

(C) 2016 SPIE · CCC code: 0277-786X/16/\$18 - doi: 10.1117/12.2240597

Proc. of SPIE Vol. $9905990501-1$ 
The papers in this volume were part of the technical conference cited on the cover and title page. Papers were selected and subject to review by the editors and conference program committee. Some conference presentations may not be available for publication. Additional papers and presentation recordings may be available online in the SPIE Digital Library at SPIEDigitallibrary.org.

The papers reflect the work and thoughts of the authors and are published herein as submitted. The publisher is not responsible for the validity of the information or for any outcomes resulting from reliance thereon.

Please use the following format to cite material from these proceedings:

Author(s), "Title of Paper," in Telescopes and Instrumentation 2016: Ultraviolet to Gamma Ray, edited by Jan-Willem A. den Herder, Tadayuki Takahashi, and Marshall Bautz, Proceedings of SPIE Vol. 9905 (SPIE, Bellingham, WA, 2016) Six-digit Article CID Number.

ISSN: 0277-786X

ISSN: 1996-756X (electronic)

ISBN: 9781510601895

ISBN: 9781510601901 (electronic)

Published by

SPIE

P.O. Box 10, Bellingham, Washington 98227-0010 USA

Telephone +1 3606763290 (Pacific Time) · Fax +1 3606471445

SPIE.org

Copyright @ 2016 , Society of Photo-Optical Instrumentation Engineers.

Copying of material in this book for internal or personal use, or for the internal or personal use of specific clients, beyond the fair use provisions granted by the U.S. Copyright Law is authorized by SPIE subject to payment of copying fees. The Transactional Reporting Service base fee for this volume is $\$ 18.00$ per article (or portion thereof), which should be paid directly to the Copyright Clearance Center (CCC), 222 Rosewood Drive, Danvers, MA 01923 . Payment may also be made electronically through CCC Online at copyright.com. Other copying for republication, resale, advertising or promotion, or any form of systematic or multiple reproduction of any material in this book is prohibited except with permission in writing from the publisher. The CCC fee code is 0277-786X/16/\$18.00.

Printed in the United States of America.

Publication of record for individual papers is online in the SPIE Digital Library.

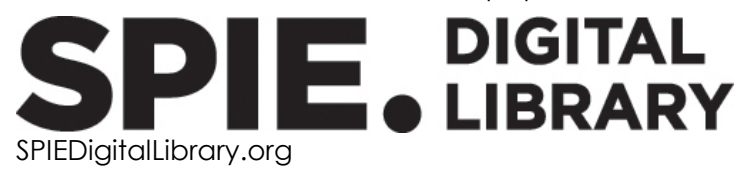

Paper Numbering: Proceedings of SPIE follow an e-First publication model. A unique citation identifier (CID) number is assigned to each article at the time of publication. Utilization of CIDs allows articles to be fully citable as soon as they are published online, and connects the same identifier to all online and print versions of the publication. SPIE uses a six-digit CID article numbering system structured as follows:

- The first four digits correspond to the SPIE volume number.

- The last two digits indicate publication order within the volume using a Base 36 numbering

system employing both numerals and letters. These two-number sets start with 00, 01, 02, 03, 04, 05, 06, 07, 08, 09, OA, OB ... 0Z, followed by 10-1Z, 20-2Z, etc. The CID Number appears on each page of the manuscript. 


\title{
Contents
}

\author{
XV Authors \\ xxxiii Conference Committee
}

\section{Part One}

SOLAR/UV I

990502 SMILE: a joint ESA/CAS mission to investigate the interaction between the solar wind and Earth's magnetosphere [9905-1]

990503 The Solar Ultraviolet Imaging Telescope onboard Aditya-L1 [9905-2]

990504 World Space Observatory Ultraviolet mission: status 2016 [9905-3]

990505 Galaxy evolution spectroscopic explorer: scientific rationale [9905-4]

990506 CHISL: the combined high-resolution and imaging spectrograph for the LUVOIR surveyor [9905-5]

SOLAR/UV II

990507 Optical design and optical properties of a VUV spectrographic imager for ICON mission [9905-6]

990508 Chromospheric LAyer SpectroPolarimeter (CLASP2) [9905-7]

990509 The Miniature X-ray Solar Spectrometer (MinXSS) CubeSats: spectrometer characterization techniques, spectrometer capabilities, and solar science objectives [9905-8]

9905 OA SISTINE: a pathfinder for FUV imaging spectroscopy on future NASA astrophysics missions [9905-9]

SOLAR/UV III

9905 OD Ultraviolet imaging detectors for the GOLD mission [9905-12]

9905 OE The FOXSI solar sounding rocket campaigns [9905-13]

9905 OF Flight production of Caliste-SO: the hard $\mathrm{x}$-ray spectrometers for solar orbiter/STIX instrument [9905-10] 
9905 0J Development of a 32-detector CdTe matrix for the SVOM ECLAIRs x/gamma camera: tests results of first flight models [9905-125]

9905 OL Fast event recognition for $\mathbf{x}$-ray silicon imagers [9905-19]

9905 OM Development of wide-field low-energy x-ray imaging detectors for Hiz-GUNDAM [9905-20]

\section{DETECTOR II}

990500 Front-end ASICs for high-energy astrophysics in space (Invited Paper) [9905-21]

$99050 Q$ Development of $\mathbf{x}$-ray microcalorimeter imaging spectrometers for the $\mathbf{X}$-ray Surveyor mission concept [9905-23]

9905 OS Toward large $\mu$-calorimeters $\mathbf{x}$-ray matrices based on metal-insulator sensors and HEMTs/SiGe cryo-electronics [9905-25]

ASTRO-H I

9905 OU The ASTRO-H (Hitomi) x-ray astronomy satellite [9905-27]

9905 OW In-flight performance of the Soft X-ray Spectrometer detector system on ASTRO-H [9905-29]

$99050 \mathrm{X}$ Vibration isolation system for cryocoolers of Soft X-ray Spectrometer (SXS) onboard ASTRO-H (Hitomi) [9905-30]

9905 OY In-orbit operation of the ASTRO-H SXS [9905-31]

$99050 Z$ First peek of ASTRO-H Soft X-ray Telescope (SXT) in-orbit performance [9905-32]

\section{ASTRO-H II}

990510 Soft x-ray imager (SXI) onboard ASTRO-H [9905-33]

990511 The hard X-ray imager (HXI) onboard ASTRO-H [9905-34]

990512 Performance of ASTRO-H hard x-ray telescope (HXT) [9905-35]

990513 The soft gamma-ray detector (SGD) onboard ASTRO-H [9905-36]

990514 ASTRO-H data analysis, processing and archive [9905-37]

POLARIZATION

990517 The Imaging X-ray Polarimetry Explorer (IXPE) [9905-40]

iv 
$99051 \mathrm{~A} \quad$ Hard x-ray imaging polarimeter for PolariS [9905-43]

\section{ASTROSAT}

9905 1D Large Area X-ray Proportional Counter (LAXPC) instrument onboard ASTROSAT [9905-45]

$99051 \mathrm{I} \quad$ In-orbit performance of SXT aboard AstroSat [9905-46]

9905 IF In-orbit performance of UVIT on ASTROSAT [9905-47]

$99051 G$ In-orbit performance AstroSat CZTI [9905-48]

NICER

$99051 \mathrm{H}$ The Neutron star Interior Composition Explorer (NICER): design and development [9905-49]

$990511 \quad$ NICER instrument detector subsystem: description and performance [9905-50]

SRG

$99051 \mathrm{~J} \quad$ Status of ART-XC/SRG instrument [9905-51]

$99051 \mathrm{~K} \quad$ eROSITA on SRG [9905-52]

NEW MISSIONS

$99051 \mathrm{~L} \quad$ Localization algorithms for micro-channel x-ray telescope on board SVOM space mission [9905-53]

$99051 \mathrm{M}$ The Transient High Energy Sky and Early Universe Surveyor (THESEUS) [9905-54]

$99051 \mathrm{~N}$ DIOS: the dark baryon exploring mission [9905-55]

9905 IR The LOFT mission concept: a status update [9905-59]

\section{OPTICS I}

9905 is Lightweight and high-resolution single crystal silicon optics for x-ray astronomy [9905-60]

$99051 \mathrm{~V}$ Development of a direct fabrication technique for full-shell x-ray optics [9905-63]

$99051 \mathrm{X}$ Critical-angle $\mathrm{x}$-ray transmission grating spectrometer with extended bandpass and resolving power $>10,000$ [9905-65] 
$9905 \mathrm{IY} \quad$ Aberrations in square pore micro-channel optics used for $\mathbf{x}$-ray lobster eye telescopes [9905-66]

990520 Development of X-ray multilayer telescope optics for XTP mission [9905-68]

990521 Progress on the fabrication of high resolution and lightweight monocrystalline silicon $\mathrm{x}$-ray mirrors [9905-69]

990522 Gas bearing slumping and figure correction of x-ray telescope mirror substrates [9905-70]

990523 Slumped glass optics development with pressure assistance [9905-71]

$990524 \quad$ New lithographic techniques for x-ray spectroscopy [9905-72]

ATHENA I

990526 ATHENA: system studies and optics accommodation [9905-74]

990527 The ATHENA optics development [9905-75]

990529 Mass production of silicon pore optics for ATHENA [9905-77]

\section{ATHENA II}

9905 2A The wide field imager instrument for ATHENA [9905-78]

9905 2B ATHENA Wide Field Imager key science drivers [9905-79]

9905 2C Studies of prototype DEPFET sensors for the wide field imager of ATHENA [9905-80]

9905 2D WFI electronics and on-board data processing [9905-81]

\section{Part Two}

9905 2E Thermal analysis of the WFI on the ATHENA observatory [9905-82]

ATHENA III

$99052 \mathrm{G} \quad \mathrm{X}-\mathrm{IFU}$ technical challenge [9905-84]

$99052 \mathrm{H} \quad$ Transition-edge sensor pixel parameter design of the microcalorimeter array for the $\mathrm{x}$-ray integral field unit on ATHENA [9905-85]

990521 The focal plane assembly for the ATHENA x-ray integral field unit instrument [9905-86] 
9905 2J Preliminary thermal architecture of the X-IFU instrument dewar [9905-87]

9905 2K The Cryogenic AntiCoincidence detector for ATHENA X-IFU: a program overview [9905-88]

GAMMA

$99052 \mathrm{M} \quad$ MeV gamma-ray observation with a well-defined point spread function based on electron tracking [9905-90]

9905 2N The e-ASTROGAM gamma-ray space mission [9905-91]

990520 The development of a low energy Compton imager for GRB polarization studies [9905-92]

$99052 P \quad$ Performance study of the gamma-ray bursts polarimeter POLAR [9905-93]

$99052 \mathrm{~F}$ First flight of the Gamma-Ray Imager/Polarimeter for Solar flares (GRIPS) instrument [9905-94]

$99052 R \quad$ First measurement of the polarisation asymmetry of a gamma-ray beam between 1.74 to $74 \mathrm{MeV}$ with the HARPO TPC [9905-95]

\section{POSTER SESSION: SOLAR/UV}

$99052 \mathrm{~T} \quad$ Correcting for errors due to walk and geometric distortion in the COS FUV detector [9905-136]

$99052 \mathrm{U}$ In-flight performance of the polarization modulator in the CLASP rocket experiment [9905-137]

$99052 \mathrm{~V}$ The re-flight of the Colorado high-resolution Echelle stellar spectrograph (CHESS): improvements, calibrations, and post-flight results [9905-138]

9905 2W Alignment and calibration of the ICON-FUV instrument: development of a vacuum UV facility [9905-140]

$99052 X \quad$ The qualification campaign of the EUI instrument of Solar Orbiter [9905-141]

9905 2Y Full-Stokes polychromatic polarimeter design for Arago [9905-142]

$99052 Z$ UVESP: ultraviolet visible Echellé spectropolarimeter for stellar astrophysics [9905-143]

990530 Simulations of the WUVS instrument [9905-144]

990531 Fireball multi object spectrograph: as-built optic performances [9905-145]

990532 An ultraviolet imager to study bright UV sources [9905-146]

990533 A near ultraviolet solar-blind telescope design using silicon CCD detectors [9905-147] 
990534 Concept study for a compact homodyne astrophysics spectrometer for exoplanets (CHASE) [9905-148]

990535 Numerical simulation of space UV spectrographs [9905-149]

990536 WSO-UV ground segment for observation optimisation [9905-150]

990537 Optical design of WUVS instrument: WSO-UV spectrographs [9905-151]

990538 Design of a wide field far-UV spectrometer for a mission to Mars [9905-152]

990539 Near UV imager with an MCP-based photon counting detector [9905-153]

9905 3B Spectral calibration of CCDs and multilayer filters intended for future space applications [9905-155]

$99053 \mathrm{C}$ A technique for selectable band imaging in the ultraviolet and optical [9905-158]

9905 3D Optical alignment of the Chromospheric Lyman-Alpha Spectro-polarimeter using sophisticated methods to minimize activities under vacuum [9905-159]

$99053 \mathrm{E} \quad$ An optimized Fresnel array for a test space mission in UV [9905-160]

9905 3F Development of a flight qualified $100 \times 100 \mathrm{~mm}$ MCP UV detector using advanced cross strip anodes and associated ASIC electronics [9905-161]

$99053 \mathrm{G}$ Ultraviolet detector with CMOS-coupled microchannel plates for future space missions [9905-162]

$99053 \mathrm{H} \quad$ Optical and structural characterization of reflective quarter wave plates for EUV range [9905-163]

990531 Design and improvements of the Attitude Control System of the FIREBall balloon experiment [9905-164]

$99053 \mathrm{~J}$ The effects of dust outbursts on the anomalistic features observed by Rosetta Alice around 67P/Churyumov-Gerasimenko [9905-165]

POSTER SESSION: ASTRO-H

$99053 \mathrm{~L}$ The design, implementation, and performance of the ASTRO-H SXS calorimeter array and anti-coincidence detector [9905-97]

$99053 \mathrm{M}$ System design and implementation of the detector assembly for the ASTRO-H soft $\mathrm{x}$-ray spectrometer [9905-98]

$99053 \mathrm{~N}$ Cryogen-free operation of the soft $\mathbf{x}$-ray spectrometer instrument [9905-99]

990530 Design and on-orbit operation of the adiabatic demagnetization refrigerator on the Hitomi soft $x$-ray spectrometer instrument [9905-100] 
9905 3P Porous plug phase separator and superfluid film flow suppression system for the soft $x$-ray spectrometer onboard ASTRO-H [9905-101]

$99053 Q$ The design, implementation, and performance of the ASTRO-H SXS aperture assembly and blocking filters [9905-102]

9905 3R Thermal analyses for initial operations of the Soft X-Ray Spectrometer (SXS) onboard ASTRO-H [9905-103]

990535 Performance of the helium dewar and cryocoolers of ASTRO-H SXS [9905-104]

9905 3T In-flight performance of pulse processing system of the ASTRO-H soft x-ray spectrometer [9905-105]

$99053 \mathrm{U} \quad$ In-flight verification of the calibration and performance of the ASTRO-H (Hitomi) soft $\mathrm{x}$-ray spectrometer [9905-106]

9905 3W Ground calibration of the ASTRO-H (Hitomi) soft x-ray spectrometer [9905-108]

$99053 X$ The ASTRO-H SXT performance to the large off-set angles [9905-110]

$99053 Y$ Reflectivity around the gold $M$-edges of $x$-ray reflector of the soft $x$-ray telescope onboard ASTRO-H [9905-111]

990532 Reflectivity around the gold L-edges of $x$-ray reflector of the soft $x$-ray telescope onboard ASTRO-H [9905-112]

990541 Ray-tracing simulation and in-orbit performance of the ASTRO-H hard x-ray telescope (HXT) [9905-1 14]

990542 A laboratory test setup to study the stability of operation of the CdTe detectors within ASTRO-H HXI [9905-115]

POSTER SESSION: DETECTORS

990544 The evolution of the ACIS contamination layer over the 16-year mission of the Chandra Xray Observatory [9905-117]

990545 Evolution of temperature-dependent charge transfer inefficiency correction for ACIS on the Chandra X-ray Observatory [9905-118]

990546 Front-end readout ASIC for charged particle counting with the RADEM instrument on the ESA JUICE mission [9905-119]

990547 Developments in calibration of EUV and VUV detectors for solar orbiter instrumentation using synchrotron radiation [9905-120]

9905 4B Development of a pixelated CdTe detector module for a hard-x and gamma-ray imaging spectrometer application [9905-124]

9905 4C Directly deposited blocking filters for high-performance silicon x-ray detectors [9905-126] 
9905 4D Line profile modelling for multi-pixel CZT detectors [9905-127]

POSTER SESSION: POLARIZATION

9905 4G Performance of the Gas Pixel Detector: an x-ray imaging polarimeter for upcoming missions of astrophysics [9905-132]

$99054 \mathrm{H}$ The on-board calibration system of the X-ray Imaging Polarimetry Explorer (XIPE) [9905-133]

\section{Part Three}

99054 Geometrical tools for the analysis of $x$-ray polarimetric signals [9905-270]

\section{POSTER SESSION: NEW MISSIONS}

9905 4L The microchannel $x$-ray telescope status [9905-217]

$99054 \mathrm{~N}$ Multi-directional measurements of high energy particles from the Sun-Earth L1 point with STEPS [9905-219]

$99054 \mathrm{P}$ The evolution of structure and feedback with Arcus [9905-221]

$99054 \mathrm{~V} \quad$ Fast simulation of the NICER instrument [9905-227]

9905 4W Ground calibration of the Silicon Drift Detectors for NICER [9905-228]

9905 4X Performance of NICER flight x-ray concentrator [9905-229]

$99054 \mathrm{Y}$ Large Observatory for $\mathrm{x}$-ray Timing (LOFT-P): a Probe-class mission concept study [9905-230]

990550 ART-XC/SRG: results of qualification thermo-vacuum tests [9905-232]

990551 Results of ground tests and calibration of x-ray focal plane detectors for ART-XC/SRG instrument [9905-233]

990552 An updated approach to the study of proton propagation in the eROSITA mirror system [9905-234]

990556 Ray-tracing critical-angle transmission gratings for the X-ray Surveyor and Explorer-size missions [9905-238]

990557 A new type of multiple image $\mathrm{x}$-ray interferometer for arcseconds and sub-arcseconds sources [9905-239]

9905 5C Compact hard $x$-ray imaging system with a large FOV [9905-269] 
9905 5D Point spread function of ASTRO-H Soft X-ray Telescope (SXT) [9905-109]

$99055 \mathrm{E} \quad$ Development of $\mathrm{x}$-ray spectroscopic polarimetry with bent Si crystals and CFRP substrate [9905-130]

POSTER SESSION: ATHENA

9905 5F Observing the WHIM with ATHENA [9905-166]

$99055 G$ Detectability of exoplanet transits with ATHENA's WFI instrument: testing for white and correlated noise [9905-167]

9905 5K X-ray mirror development and testing for the ATHENA mission [9905-171]

$99055 \mathrm{~N} \quad$ New $x$-ray parallel beam facility XPBF 2.0 for the characterization of silicon pore optics [9905-174]

990550 Simulation and modeling of silicon pore optics for the ATHENA x-ray telescope [9905-176]

9905 5P Development and production of a multilayer-coated x-ray reflecting stack for the ATHENA mission [9905-177]

$99055 R \quad$ Optimising the multiplex factor of the frequency domain multiplexed readout of the TESbased microcalorimeter imaging array for the X-IFU instrument on the ATHENA X-ray observatory [9905-179]

$99055 S$ Development of frequency domain multiplexing for the X-ray Integral Field unit (X-IFU) on the ATHENA [9905-180]

$99055 \mathrm{~T}$ The impact of crosstalk in the X-IFU instrument on ATHENA science cases [9905-181]

$99055 \mathrm{U} \quad$ Gain drift compensation with no-feedback-loop developed for the X-IFU/ATHENA readout chain [9905-182]

9905 5V Microcalorimeter pulse analysis by means of principle component decomposition [9905-183]

9905 5W Performance assessment of different pulse reconstruction algorithms for the ATHENA X-ray Integral Field Unit [9905-184]

$99055 X \quad$ The Cryogenic Anti-Coincidence detector for ATHENA X-IFU: pulse analysis of the AC-S7 single pixel prototype [9905-185]

$99055 Y$ The mechanical and EM simulations of the CryoAC for the ATHENA X-IFU [9905-186]

$99055 Z$ The new cryogenic silicon monolithic micro-bridged Anticoincidence detector for the XIFU of ATHENA [9905-187]

990560 Temperature effects on the performances of the ATHENA X-IFU thermal filters [9905-188] 
990561 The filter wheel and filters development for the X-IFU instruments onboard ATHENA [9905-189]

990562 Conceptual design of the X-IFU Instrument Control Unit on board the ESA ATHENA mission [9905-190]

990563 Updates on the background estimates for the X-IFU instrument onboard of the ATHENA mission [9905-191]

990564 TESSIM: a simulator for the ATHENA-X-IFU [9905-192]

990565 Background studies for ATHENA: status of the activities at IAAT [9905-193]

990566 Surface investigation and aluminum oxide estimation on test filters for the ATHENA X-IFU and WFI detectors [9905-194]

990567 Spectroscopic performance of DEPFET active pixel sensor prototypes suitable for the high count rate ATHENA WFI detector [9905-195]

990568 The filter and calibration wheel for the ATHENA wide field imager [9905-196]

\section{POSTER SESSION: GAMMA}

9905 6B Ex luna, scientia: Iunar occultation as a paradigm for nuclear astrophysics [9905-197]

9905 6C GAMCOTE: a prototype for an advanced Compton Telescope [9905-198]

9905 6D Experimental verification of the HERD prototype at CERN SPS [9905-199]

9905 6E PANGU: a wide field gamma-ray imager and polarimeter [9905-200]

9905 6G Gamma ray spectrometer for future Mars mission: design concept and simulation study [9905-202]

990561 A compact and modular x- and gamma-ray detector with a Csl scintillator and doublereadout Silicon Drift Detectors [9905-204]

9905 6J Characterization of a LaBr3 scintillator with multi-cell Silicon Drift Detector (SDD) readout [9905-205]

$99056 \mathrm{~K}$ The Advanced Scintillator Compton Telescope (ASCOT) balloon project [9905-206]

$99056 \mathrm{~L}$ A concept for a soft gamma-ray concentrator using thin-film multilayer structures [9905-207]

$99056 \mathrm{P}$ In-depth calibration of a Lave lens prototype composed of Fe and Al mosaic crystals [9905-214] 
POSTER SESSION: OPTICS

$99056 Q \quad$ Multilayer coating of XTP telescope mirrors [9905-244]

$99056 \mathrm{R} \quad \mathrm{X}$-ray mirror module analytical design from field of view requirement and stray light tolerances [9905-245]

$99056 \mathrm{~V}$ Development of low-stress Iridium coatings for astronomical x-ray mirrors [9905-249]

9905 6W Monte Carlo simulations of soft proton flares: testing the physics with XMM-Newton [9905-250]

$99056 \mathrm{X}$ Alignment and distortion-free integration of lightweight mirrors into meta-shells for highresolution astronomical $x$-ray optics [9905-251]

$99056 Y \quad$ Indirect slumping of D263 glass on Fused Silica mould [9905-252]

990570 Analysis on the use of vacuum oven for the indirect slumping of glass $x$-ray mirror segments [9905-254]

990571 Development of an x-ray telescope using the carbon fiber reinforced plastic [9905-255]

990573 On the alignment and focusing of the Marshall Grazing Incidence X-ray Spectrometer (MaGIXS) [9905-257]

990574 Design of a medium size x-ray mirror module based on thin glass foils [9905-258]

990576 Simulating x-ray telescopes with McXtrace: a case study of ATHENA's optics [9905-260]

990578 Ion beam figuring of thin glass plates: achievements and perspectives [9905-262]

$99057 \mathrm{~A}$ Design and analysis of an x-ray mirror assembly using the meta-shell approach [9905-264]

$99057 \mathrm{E}$ Development of manufacture of mirror glass substrate for $\mathbf{x}$-ray timing and polarization observatory [9905-268] 
Proc. of SPIE Vol. $9905990501-14$

Downloaded From: https://www.spiedigitallibrary.org/conference-proceedings-of-spie on 26 Apr 2023 Terms of Use: https://www.spiedigitallibrary.org/terms-of-use 


\section{Authors}

Numbers in the index correspond to the last two digits of the six-digit citation identifier (CID) article numbering system used in Proceedings of SPIE. The first four digits reflect the volume number. Base 36 numbering is employed for the last two digits and indicates the order of articles within the volume. Numbers start with 00, 01, 02, 03, 04, 05, 06, 07, 08, 09, OA, OB...0Z, followed by 10-12, 20-2Z, etc.

\author{
Aboudan, A., 2N \\ Ackermann, Jörg, 46 \\ Adams, Joseph S., 0Q, 2H, 3L, 3M, 3Q, 3W, 5S \\ Adhyaru, P. R., 4N \\ Adkins, Phillip W., 1H \\ Agrawal, P. C., 1D \\ Agudo, I., IR \\ Ahangarianabhari, M., IR \\ Aharonian, Felix, OU \\ Aird, James, 2B \\ Akamatsu, Hiroki, OU, OY, 2H, 2I, 5R, 5S, 5V \\ Ake, Thomas, 2T \\ Akimoto, Fumie, oU \\ Akimov, Valery, 1 J, 51 \\ Al Matroushi, Hessa Rashid, 38 \\ Albergo, Sebastiano, 6D \\ Albert, Cheryl L., 1H \\ Albertus, C., IR \\ Albrecht, Sebastian, 2D \\ Alford, M., IR \\ Aliotta, Paul H., 6L \\ Allen, Steve, OU \\ Allgood, Kim D., 21 \\ Allured, Ryan, 4P, 73 \\ Alpar, A., 1R \\ Altamirano, D., 1R \\ Altan, Mehmet Akif, 46 \\ Álvarez, J.-M., 4B \\ Álvarez, Laura, 1R, 4B, 4Y \\ Amano, S., 2R \\ Amati, Lorenzo, 1M, 1R, 61 \\ Ambily, S., 39 \\ Ambroglini, Filippo, 6D \\ Ambrosi, Giovanni, 6D, 6E \\ Amman, M., 2Q \\ Amoros, C., 0J, $1 R$ \\ Anabuki, Naohisa, 0U, 10 \\ Anders, John F., $1 \mathrm{H}$ \\ Anderson, Tyler, OL \\ Andersson, N., IR \\ André, Jérome, 2J \\ Andritschke, Robert, 1K, 2C, 67 \\ Angelini, Lorella, OU, 14 \\ Antia, H. M., ID \\ Antonelli, A., $1 R$ \\ Arefiev, V., $1 \mathrm{~J}$ \\ Argan, A., 1R, 2I, 2K, 2N, 5X \\ Arimoto, Makoto, $\mathrm{OM}$ \\ Arnaud, Keith, OU
}

Arnold, W., IV

Arsenovic, Petar, 3Q

Artigue, R., 1R

Artigues, B., 1R, 4B

Arzoumanian, Zaven, 1H, 11, 4V, 4W, 4X

Asai, Makoto, OU

Asensio Ramos, Andres, 08

Atkins, C., IV

Atteia, J.-L., OJ, 1R

Attié, D., 2R

Aubaret, K., OJ

Auchère, Frédéric, 08, 2X, 3D

Audard, Marc, OU, $3 \mathrm{U}$

Awaki, Hisamitsu, OU, 12, 41, 71

Axelsson, Magnus, OU

Ayers, Travis, $3 Q$

Aylward, Andrew T., $1 \mathrm{H}$

Ayre, M., 26

Azman, Suleyman, 46

Aznar Cuadrado, R., 2X

Azzarello, Philipp, 0U, 1R, 6D, 6E

Babazaki, Y., IN

Babyshkin, Vladimir, 1 J, 1K

Bähr, Alexander, 2C, 67

Bai, Yonglin, 6D

Bai, Yuhong, 6Q, 7E

Bain, H. M., 2Q

Bakala, P., IR

Baker, Charles L., 1H

Baldazzi, G., 1R, 6I, 6J

Baldini, Luca, 4G, 6D

Baldo, M., IR

Ballantyne, D., 1R

Balman, S., 1R

Balsamo, Erin R., 1H, 4X

Baluta, Chris, OU

Bamba, Aya, OU, 10

Bamford, William A., $1 \mathrm{H}$

Bancroft, Christopher M., 6K

Bandler, Simon R., OQ, 2H, 2I, 5S, 5T, 5W, 64

Bando, Nobutaka, OU

Bando, Takamasa, 2U, 3D

Banerjee, D., 6G

Banerjee, Dipankar, 03

Banerjee, S. B., $4 \mathrm{~N}$

Bao, Tianwei, 2P, 6D

Bapat, B., $4 \mathrm{~N}$

Barbera, Marco, 1R, 21, 60, 61, 66, 68

Barcons, Xavier, 5F, 5W 
Barret, Didier, 1R, 2H, 2I, 5F, 5T, 5W, 61

Barrière, Nicolas, 27, 5N, 5P, 6P

Barve, Indrajit $V_{\text {., }} 1 \mathrm{~F}$

Basargina, $0 ., 36$

Basile, John, OX

Basso, Stefano, 23, 74, 78

Batsch, T., 2P

Battiston, Roberto, 6D

Baumgartner, Wayne H., 1I, 4W

Bautz, Marshall W., OL, OU, 45, 4C, 4P, 56

Bavdaz, Marcos, 26, 27, 29, 5N, 50

Bayer, Jörg, 2D

Baykal, A., 1R

Baysinger, Michael, $4 Y$

Beabout, Brent L., $2 U$

Beabout, Dyana L., 2U

Beardmore, Andy P., $1 \mathrm{E}$

Becker, Chris, $4 Y$

Becker, Dan, OQ

Becker, Werner, $1 \mathrm{~K}$

Bednarzik, Martin, OF

Begelman, M., IR

Behar, E., IR

Behar, O., IR

Beillimaz, C., 5U

Bellazzini, Ronaldo, 17, 4G, 4H

Belloni, T., IR

Bellutti, P., 6I, 6J

Belluzzi, Luca, 08

Benegalrao, Suyog S., $1 \mathrm{H}$

Bennett, Douglas, OQ, 2H

Berghmans, D., $2 X$

Bernard, D., 2N, 2R

Bernardini, F., $1 \mathrm{R}$

Bernardini, Paolo, 6D

Bernasconi, T., 2P

Berry, Daniel L., 1H

Bertoli, W., 6C

Bertuccio, G., $1 R$

Betancourt-Martinez, Gabriele, 0Q, 2H

Bhalerao, Varun B., 1G, 4D

Bhalwani, Shiraz, $1 \mathrm{H}$

Bhatia, Ritwik, $1 \mathrm{X}$

Bhattacharya, Dipankar, 1G, 4D

Bhattacharyya, Sudip, 1E, IR

Bialas, Thomas G., OU, OY, 3N, 30

Bianchi, Davide, 2C

Bianchi, S., IR

Bianchini, A., 1R

Biasotti, M., 2K, 5X, 5Y, $5 Z$

Billot, Marc, OF, OJ

Binko, P., IR

Birrer, Guy, OF

Biskach, Michael P., 1S, 21, 6X

Black, J. Kevin, $1 \mathrm{H}$

Blain, Dominique, $\mathrm{OF}$

Blain, Pascal, 2W

Blandford, Roger, OU, 13

Blaurock, Carl, $1 \mathrm{H}$

Blay, P., IR
Blondel, Claire, OF

Bloser, Peter F., 2O, 6K, 6L

Bocchino, F., IR

Bode, M., IR

Bodin, P., IR

Bogdan, Akos, 44

Boggs, Steven E., 2Q, 6P

Boller, Thomas, $1 \mathrm{~K}$

Bombaci, I., IR

Bonafede, Joseph A., 7A

Bonino, D., 62

Bonnet Bidaud, J.-M., IR

Bookbinder, J., 4P

Bordon, S., OJ

Borghi, G., 6J

Bornemann, Walter, $1 \mathrm{~K}$

Böttcher, M., 6E

Boussadia, Mohamed, OF

Boutloukos, S., 1 R

Bouyjou, F., IR

Boyce, Kevin R., OU, OW, OY, 3N, 3O, 3T, 3U, 3W

Bozzo, Enrico, 1R, 4H, 4Y, 61

Bradley, L., IR

Braga, J., IR

Brand, Thorsten, 5F, 5T, 5W, 64

Brandt, Soren, 1R, 2D, 4Y

Branduardi-Raymont, Graziella, 02, 61

Bräuninger, Heinrich, 1K

Bray, Nicolas, 3l

Bregman, J., 4P

Brejnholt, Nicolai F., 3Z, 6P

Brekosky, Regis P., 3L

Brenneman, Laura W., OU, 4P

Breunig, Elias, 6Y, 70

Brez, Alessandro, 4G

Brickhouse, N., 4P

Briggs, M. S., IR

Britvitch, I., 2P

Brogna, A., 2N

Bronke, Ginger M., $1 \mathrm{H}$

Brown, E., IR

Brown, Gary L., 1H

Brown, Gregory V., OU, OY, 3U, 3W

Bruccoleri, Alexander R., 1X, 73

Bruel, P., 2R

Bruijn, Marcel P., 2I, $5 S$

Brulin, G., 6C

Brunner, Hermann, $1 \mathrm{~K}$

Buballa, M., IR

Bucciantini, N., IR

Budinoff, Jason G., $1 \mathrm{H}$

Budtz-Jørgensen, C., 1R

Buitrago-Casas, Juan Camilo, OE

Bulbul, Esra, OU

Bulgarelli, Andrea, 2K, 2N, 63, 6W

Buntov, M., 1J, 50

Burderi, L., IR, 6J

Burgay, M., IR

Burkert, Wolfgang, $1 \mathrm{~K}$

Burrows, David N., OL, 4P 
Bursa, M., IR

Burwitz, Vadim, 1K, 27, 4H, 4P, 6Y

Bykov, A., 2N

Cabruja, E., 4B

Cackett, E., IR

Cackett, Edward, OU

Cadoux, F., 1R, 2P

Cais, P., $1 R$

Caliandro, G. A., IR

Calvet, D., 2R

Campana, R., 1R, 2N, 4I, 6I, 6J

Campana, S., $1 R$

Canavan, Edgar R., 0U, 30

Cantwell, Jeffrey D., $1 \mathrm{H}$

Cao, X., IR

Capitanio, F., $1 \mathrm{R}$

Capobianco, V., 62

Caraveo, P., $2 \mathrm{~N}$

Cardillo, M., 2N

Carlsson, Mattias J. L., 08

Carpano, Stefania, 5G

Carson, Billy, $4 \mathrm{Y}$

Casares, J., IR

Casella, P., IR

Caspi, Amir, 09, 2Q

Castro Tirado, Alberto J., 1R, 4H

Cavazzuti, E., 1R, 2K

Cavechi, Y., IR

Cazeau, Thoniel, $1 \mathrm{H}$

Ceballos, Maria Teresa, 5F, 5W, 64

Celestin, S., IR

Cerda-Duran, P., IR

Cernuda, I., 2P

Chaffin, Michael, 38

Chai, J. Y., 2P

Chakrabarty, D., $4 \mathrm{~N}$

Chakrabarty, Deepto, 1R, 4Y

Chalifoux, Brandon, 22

Chamel, N., IR

Champey, Patrick, 73

Chan, Kai-Wing, 1S, 6X

Chandra, Sunil, $1 \mathrm{E}$

Chang, Bill, $\mathrm{OZ}$

Chang, J., 6E

Chapman, Jack, 4Y

Charles, Ivan, 2J

Charvolin, T., OS

Château, F., IR

Chatterjee, Subhamoy, 03

Chattopadhyay, T., 4D

Chattopadhyay, Tanmoy, $1 \mathrm{G}$

Chaudhuri, Saptarshi, OQ

Chauhan, Jai Verdhan, ID

Cheimets, Peter, 73

Chen, C., IR

Chen, Philip T., $1 \mathrm{H}$

Chen, S., $5 \mathrm{U}$

Chen, Y., IR

Chen, $Y ., 1 R$

Chen, Zhen, 6D
Chenevez, J., $1 \mathrm{R}$

Chernyakova, Maria, OU, IR, 6E

Chervenak, James A., OQ, 2H, 2I, 3L, 5S

Chiao, Meng P., OU, OW, OX, OY, 2H, 3L, 3M, 3N,

$3 Q, 3 S, 3 T, 3 U, 3 W$

Chitnis, Varsha, $1 \mathrm{E}$

Chmeissani, M., 4B

Chordia, Pravin, 03

Chou, Cathy, 07, 2W

Christe, Steven, OE

Christensen, Finn E., 27, 3Z, 5K, 5O, 5P, 76

Churazov, Eugene, $1 \mathrm{~J}, 1 \mathrm{~K}$

Ciaravella, Angela, 60

Cibik, Levent, 5K, 5N, 5P

Cirtain, Jonathan W., 08, 73

Civitani, Marta, 23, 50, 74, 78

Clement, Thomas G., $1 \mathrm{H}$

Clerc, Nicolas, $1 \mathrm{~K}$

Clermont, Lionel, $2 \mathrm{~W}$

Cobo, Beatriz, 5F, 5W, 64

Coker, J., IR

Colangelo, Andrew T., $1 \mathrm{H}$

Colas, P., 2R

Cole, R., IR

Coleman, Jerry S., $1 \mathrm{H}$

Collon, Maximilien, 27, 29, 5N, 5P

Collura, Alfonso, 1R, 60

Comastri, Andrea, 2B

Conconi, P., 50

Coopersmith, Jonathan D., $1 \mathrm{H}$

Coppi, Paolo, OU, 2N

Corcione, L., 62

Cordier, B., OJ

Coriat, M., IR

Cornelisse, R., 1R

Corsini, D., 2K, 5X, 5Y, $5 Z$

Corso, A. J., 3H

Costa, Enrico, 17, 4G, 4H, 4I

Costamante, L., 1R

Costantini, Elisa, OU, OY, 4P

Courtade, Sasha, OE, 38

Coutinho, Diogo, 1K

Cros, A., IR

Cui, W., IR

Cumming, A., $1 R$

Cumming, Harley, 3F

Curtis, David, 38

Curtis, T., OD

Cusumano, G., $1 R$

Czerny, B., IR

D'Aì, A., 1R, 63

Dai, Lei, 02

Dai, Ying, 20

Dai, Z., IR

D'Alessandro, Raffaello, 6D

D'Ammando, F., $1 \mathrm{R}$

D'Anca, Fabio, 68

D'Andrea, M., 2K, 5X, 5Z, 63

Daniel, Christophe, 2J

Daté, $S$., $2 R$ 
Datesman, Aaron M., OQ

Dauser, Thomas, 2B, 5F, 5T, 5W, 64

Davis, Michael, 3J

De Angelis, A., 2N

Deasy, Kieran, 5C

Dedhia, Dhiraj, 1D

Degenaar, N., 1R

De Gerone, M., 5 Y, $5 Z$

Dehaven, William E., $1 \mathrm{H}$

Deighan, Justin, 38

de la Broïse, X., OS

Delaigue, S., OJ

de la Rie, R., IR

Delbart, A., 2R

Del Hoyo, Javier, OA

D'Elia, V., IR

Della Monica Ferreira, Desiree, 27, 5K, 5O, 5P, 76

De Luca, A., IR

De Martino, D., $1 R$

De Mitri, Ivan, 6D

Del Monte, Ettore, 1R, 4G, 4H, 4I

del Pino Aleman, Tanausu, 08

Del Santo, M., IR

den Hartog, Roland, 2H, 2I, 2J, 5F, 5R, 5T, 5W, 64

den Herder, Jan-Willem A., OU, OY, 1R, 2I, 3U, 3W,

$5 \mathrm{~F}, 5 \mathrm{~T}, 5 \mathrm{~W}, 61,64$

Dennerl, Konrad, $1 \mathrm{~K}$

De Pasquale, M., $1 \mathrm{R}$

de Plaa, Jelle, 0U, 5W, 64

De Pontieu, Bart, 08

Dercksen, J. P. C., 1R, 21

DeRoo, Casey, 24, 4P

De Rosa, A., IR

DeRosa, Gisella, 2T

de Séréville, N., 6C

Desselle, Richard, $2 \mathrm{~W}$

de Vries, Cor P., OU, OY, 3U, 3W, 5S, 5V

Dewangan, Gulab Chand, 1E, $1 \mathrm{G}$

Dezalay, J.-P., OJ

Di Cosimo, S., $1 R$

Diebold, S., 1R, 52, 65

Diehl, R., 2N

Di Lalla, Niccolò, $4 \mathrm{G}$

DiPirro, Michael J., OU, OY, 3N, 3O, 3P, 3Q, 3R, 3S

Di Salvo, T., 1R

Döhring, Thorsten, 6V

Dominguez, Alexandra, 4Y

Domken, Isabelle, 2W

Donati, Modeste, OF

Done, Chris, OU

Dong, Q., OS

Dong, Yongwei, 1R, 2P, 6D

Donnarumma, I., 1R, 2N

Donovan, Eric, 02

Doriese, William B., OQ, $2 \mathrm{H}$

Dormard, J.-J., 6C

Doroshenko, V., IR

Dosme, N., 6C

Dotani, Tadayasu, OU, 10, 1A

Doty, John P., OU, 10, 1H, 11, 4V
Doumayrou, E., 4L

Doyle, G., IR

Drake, S. A., IR

Drozdova, T., $1 \mathrm{~J}$

Drumm, Paul, 2D

Duband, Lionel, 2J

Dumaye, Luc, OF

Dumesnil, C., $2 X$

Duncan, Nicole, $2 Q$

Durant, M., IR

Duranti, Matteo, 6D

Durham, R. Nick, 45

D'Urso, Domenico, 6D

Dutka, Michael, 14

Duval, Jean-Marc, 2J

Dwelly, Tom, $1 \mathrm{~K}$

Ebisawa, Ken, OU

Echt, Olof, 6L

Eckart, Megan E., OQ, OU, OW, OX, OY, 2H, 3L, 3N, $3 Q, 3 S, 3 T, 3 U, 3 W$

Edelstein, Jerry, 38

Eder, Josef, 1K, 2A, 68

Egan, Mark D., 1H, 11

Eggen, Joseph, 14

Elmi, I., 6l, 6J

Elsner, Ronald, 17, $1 \mathrm{~J}$

Ely, Justin, 2T

Emberger, Valentin, $1 \mathrm{~K}$

Emmanoulopoulos, D., 1R

England, Scott, 38

Enno, Greg, 02

Enoto, T., IR

Enoto, Teruaki, OU, 11, 13, 1H, 4X

Eraerds, Tanja, 2A

Erickson, Nicholas, 2V

Erkut, M. H., IR

Escoubet, Philippe, 02

Esposito, P., IR

Evangelista, Yuri, 1R, 4G, 6l, 6J

Evrard, Jean, 3l

Ezoe, Yuichiro, OU, OY, 1N, 3P, 3R, 3S

Fabian, Andy C., OU, 1R, 2B

Fabiani, S., 6J

Fabisinski, Leo, $4 \mathrm{Y}$

Fairbend, R., $1 Y$

Falanga, M., IR

Falcone, Abraham D., OL

Fan, Terry W.-M., $1 \mathrm{H}$

Fan, Y., 6E

Farnier, C., 6E

Fatkhullin, Timur, 35, 37

Faul, T., 6C

Favre, Y., 1R

Feldman, C. H., IR, IY

Fender, R., IR

Feng, Hua, 1R, 4H

Feroci, Marco, 1R, 4Y, 6l, 6J

Ferrari, V., $1 \mathrm{R}$

Ferreira, I., 26

Ferrigno, Carlo, OU, $1 \mathrm{R}$

xviii 
Ferro, Deneen M., 1H

Fiant, Nicolas, OF

Ficorella, F., 6l, 6J

Figueroa-Feliciano, Enectali, $3 \mathrm{~L}$

Fillingim, Matthew, 38

Finger, M. H., IR

Finger, M., $1 R$

Finkbeiner, Fred M., OQ, $2 \mathrm{H}$

Fioretti, Valentina, 2K, 2N, 63, 6W

Fiorini, M., 2K, 61

Fischer, Debra, 34

Fitzsimmons, Sean, 4X

Fleming, Brian T., 06, 0A, 2V

Foster, Adam, OU, 4P

Foster, Natalie, OE

Foster, Richard, 1H, $1 \mathrm{I}$

Fowler, Joseph W., OQ, $2 \mathrm{H}$

France, Kevin, 06, 0A, 2V, 3C

Fransen, Sebastiaan, 26, 27

Fraser, G. W., 1R

Fratter, Isabelle, OF

Frericks, M., 1R

Frey, Harald U., 07, 2W

Freyberg, Michael, 1K, 52

Friedrich, Peter, 1K, 6Y, 70

Frontera, F., 61

Frotin, M., 2R

Fujimoto, Ryuichi, OU, OW, OX, OY, 3N, 3O, 3P, 3Q, 3R, 3S, 3U, 3W

Fukazawa, Yasushi, OU, 11, 13

Fullekrug, M., 1R

Fürmetz, Maria, 1K, 2E

Furuzawa, Akihiro, 0U, 0Z, 12, 3U, 3Y, 41

Fuschino, F., 1R, 6I, 6J

Fusco, Piergiorgio, 6D

Gaballah, A. E. H., 3H

Gabler, M., 1R

Galassi, Nicholas M., $1 \mathrm{H}$

Galeazzi, Massimiliano, OU, 3L

Galliano, M., OJ

Gallo, Luigi, OU

Gallo, Luis D., 1H

Galloway, D. K., 1R

Gálvez Sanchez, J. L., 1R, 4B

Gandhi, Poshak, OU, 1R

Gandorfer, Achim M., 03

Gangemi, G. C., 6J

Gangl, Bert, $4 Y$

Gao, Jiarui, 6D

Gao, Xiaohui, 6D

Gao, Z., 1 R

Garcia, Jay, 4Y

Garcia-Berro, E., 1R

Gard, Johnathan D., OQ

Gargano, Fabio, 6D, 6E

Garnaes, Jørgen, 5K

Gaskin, Jessica A., $1 \mathrm{X}$

Gastaldello, F., 63

Gatti, F., 2I, 2K, 5X, 5Y, $5 Z$

Gauvin, N., 2P
Gburek, Szymon, 4H

Ge, Bing, 7E

Geerebaert, Y., 2R

Gendre, B., IR

Gendreau, Keith C., 1H, 11, 4V, 4W, 4X

Genolet, L., 61

Genolini, B., 6C

Georgakakis, Antonis, 1K, 2B

George, Koshy, $1 \mathrm{~F}$

Germain, Gregg, 44

Gevin, Olivier, OF, 0J, 0O, 1R

Gezari, S., IR

Gheorghe, Codin, 46

Ghigo, M., 23, 78

Ghosh, Avyarthana, 03

Ghosh, Swarna K., $1 F$

Gibelin, L., 6C

Giebels, B., 2R

Giglietto, Nicola, 6D

Giles, A. B., 1R

Gilfanov, Marat, 1 J, 1K, 1R

Gilmore, Kirk, OU

Giommi, P., 1R

Giono, Gabriel, 2U, 3D

Giovannini, G., 1R

Girish, V., 1F

Giroletti, M., 1R

Girou, D. A., 5P

Giuliani, A., 2N

Giustini, Margherita, OU

Glesener, Lindsay, OE

Glushenko, A., $1 \mathrm{~J}$

Godbole, N. M., 2Q

Godet, O., 0J

Gogus, E., IR

Goldsten, John O., 6B

Goldwurm, Andrea, 0U, 13, 1R, 5U

Golub, Leon, 73

Goluchová, K., 1R

Gomes, Albert, 31

Gómez de Castro, Ana Inés, 04, 2Z, 30

Gonzalez, F., OJ

Goodwin, P., 3M

Goosmann, Rene, 4H

Gosset, L., 1L

Gostojić, A., 6C

Gottardi, Luciano, 2H, 2I, 5R, 5S, 5V

Gottwald, Alexander, 47

Göłz, Diego, 1L, 1M, 1R, 2R, 4L

Gou, L., IR

Gouiffes, C., 1R

Goyal, S. K., 4N, 6G

Grandi, P., $1 R$

Grange, Robert, 31, 31

Grant, Catherine E., 45, 4P

Grant, Dave, 6B

Grassi, M., 1R

Grave, X., 6C

Grebenev, S., $1 \mathrm{~J}$

Green, Chris M., $1 \mathrm{H}$ 
Green, James C., OA, 2V, 3C

Grein, Christoph, 3L

Greiner, J., 1R

Grenier, I., 2N, 6E

Griffith, C., 1V

Griffith, Christopher, 4Y

Grimm, Oliver, OF

Grinberg, V., IR

Groot, P., 1R

Gros, P., 2R

Grosh, Dave, 1H

Grossberger, Christoph, 1K

Grove, J. E., 2N

Gschwender, M., 1R

Gu, Liyi, OU

Guainazzi, Matteo, 0U, 3U

Gualtieri, L., 1R

Gubarev, Mikhail, OE, 1 J, 1V

Güdel, Manuel, 1R, 2B

Guidorzi, C., 1R

Guillemot, Ph., 0J

Guizzo, G.P., 62

Gunji, Shuichi, 1A

Günther, Hans M., 56

Guy, L., 1 R

Guzman, A., 65

Gyo, M., 2X

Ha, Kong Q., $1 \mathrm{H}$

Haas, Daniel, OU, OY, 1R, 2I, 3U, 3W, 5R

Haba, Yoshito, OU, 12, 41

Haberl, Frank, $1 \mathrm{~K}$

Haensel, P., 1 R

Hagino, Kouichi, OU, 11, 13

Hahne, Devin, $\mathrm{OZ}$

Hailey, M., $1 R$

Hajdas, W., 2P, $6 \mathrm{E}$

Halain, J.-P., $2 X$

Hälker, Olaf, 1K, 2D

Hamadache, C., 2N, 6C

Hamaguchi, Kenji, OU

Hamden, E., 31

Hamuguchi, K., $1 \mathrm{R}$

Haneveld, Jeroen, 27

Hanlon, Lorraine, 2N, 6P

Hansen, F., IR

Hara, H., 3D

Harayama, Atsushi, OM, OU, 11, 5C

Harmoul, Abdullah Saif, 38

Harra, L., 2X

Harrus, llana, OU, 14

Hartmann, D. H., 1R, 2N

Hartner, Gisela, $1 \mathrm{~K}$

Hartz, Leslie, 3N

Hasanbegovic, Amir, 46

Hasegawa, Takashi, OM

Hashimoto, S., 2R

Hasouneh, Monther A., $1 \mathrm{H}$

Haswell, C. A., 1R

Hatori, Satoshi, OM

Hatsukade, Isamu, 0U, 10
Hayashi, Katsuhiro, 0U, 11, 13

Hayashi, T., IN

Hayashi, Takayuki, OU, 0Z, 12, 3U, 3X, 3Y, 3Z, 41,

5D

Hayashida, Kiyoshi, OU, 10, 1A, 57

He, J. J., $2 \mathrm{P}$

$\mathrm{He}$, Pengfei, 20

Heap, Sara, 05

Hebeler, K., IR

Heefner, Kristofer B., $1 \mathrm{H}$

Heger, A., IR

Heilmann, Ralf K., 1X, 22, 4P, 56, 73

Hempel, M., IR

Hermans, A., $2 X$

Hermsen, W., IR

Hernanz, Margarita, 1R, 2N, 4B, 4Y

Hertz, Edward, 73

Hestnes, Phyllis, $1 \mathrm{H}$

Hickman, Robert, 4Y

Hikida, Reina, $3 G$

Hill, Robert S., 14

Hilton, Gene C., OQ, 2H

Hiraga, Junko S., OU, 10

Hirose, Kazuyuki, OU

Hoadley, Keri, 06, 2V

Hoberman, Jane, $\mathrm{OE}, 2 \mathrm{Q}$

Hobson, C. W., 3M

Hoge, Lisa J., $1 \mathrm{H}$

Holland, Andrew, 02

Hołyszko, J., 23, 78

Homan, J., IR

Hopkins, Randall, $4 Y$

Horan, D., 2R

Horeau, Benoit, 42

Hornschemeier, Ann, OU

Hornstrup, A., IR

Horyani, Mihaly, 3J

Hoshino, Akio, OU, OY, 35

Hosseini, Sona, 34

Houret, B., OJ

$\mathrm{Hu}$, Bingliang, 6D

Huang, Qiushi, 20, 6Q

Huber, Alan, 11

Hudec, R., IR

Huenemoerder, David P., 4P, 56

Hughes, Eli, OL

Hughes, John, OU

Hui, Michelle, 4Y

Hull, J., OD

Hull, Tony, 05

Huovelin, J., IR

Huppenkothen, D., IR

Hurford, G. J., 2Q

Hutchings, John, $1 \mathrm{~F}$

Ichinohe, Yuto, OU, 13

lizuka, Ryo, OU, 0Z, 12, 3U, 3X, 3Y, 3Z, 41, 5D, 5E

Ikeda, Hirokazu, OM

Ikeyama, Yuki, $1 \mathrm{~A}$

Immel, Thomas J., 07

Ina, Masao, OM 
Inam, S. C., IR

Ingram, A., IR

Ingram, Luster, $4 Y$

Inove, Hajime, OU

Inove, Shota, 57

Inove, Yoshiyuki, OU

In't Zand, J. J. M., IR

Irwin, Kent D., OQ, 2H

Isern, J., $2 \mathrm{~N}$

Ishibashi, Kazunori, OU, 0Z, 12, 3Y, 41

Ishida, Manabu, OU, OZ, 12, 3U, 3X, 3Y, 3Z, 41, 5D

Ishikawa, Kumi, OU, OY, 3P, 3R, 3S

Ishikawa, Ryoko, 08, 2U, 3D

Ishikawa, Shin-nosuke, 08, OE, 2U, 3D

Ishimura, Kosei, OU, OX

Ishisaki, Yoshitaka, OU, OW, OY, 1N, 3N, 3R, 3S, 3T, 3U, 3W

Israel, G., IR

Itoh, Masayuki, oU, 12, 41

Iwai, Masachika, 10

Iwasawa, K., IR

Iwata, Naoko, OU, OX

Iyomoto, Naoko, OU, OY

Izumiya, Takanori, 5E

Izzo, L., IR

Jackson, Brian D., 2H, 2I, 5R, 5S, $5 T$

Jacobs, H. M., 1R

Jacobs, Tawanda M., $1 \mathrm{H}$

Jacques, L., $2 X$

Jacquey, C., 63

Jakobsen, Anders C., $5 \mathrm{~K}$

James, Bryan L., 30

Janardhan, P., 4N

Jelinsky, S., OD

Jenke, Peter, IR, $4 \mathrm{Y}$

Jetter, F., IR

Jewell, Chris, OU

Jhabvala, Christine A., 3L

Jiang, Li, 6Q

Jimenez Escobar, Antonio, 60

Jin, Xin, 7E

Jin, $Y .$, OS

Jing, Li, 02

Johannsen, T., $1 R$

Johansen, Tor Magnus, 46

Jonker, P., IR

Jørgensen, John L., $1 \mathrm{H}$

Josè, J., IR

Kaaret, P., IR

Kaastra, Jelle, OU, 4P, 5F

Kagawa, Yasuaki, OM

Kaiser, Michael A., 1H

Kalamkar, K., IR

Kalemci, E., IR

Kallman, Timothy, OU

Kamae, Tuneyoshi, OU

Kamath, P. U., IF

Kamble, Nilima, $1 \mathrm{E}$

Kamisiński, T., 68

Kamitsukasa, Fumiyoshi, 1A
Kanao, Kenichi, OX, 3P, 3S

Kanbach, G., 1R, 2N

Kane, Robert, 0A, 2V

Kanev, Evgeny, 35, 36

Kano, Ryouhei, 08, 2U, 3D

Kara, Erin, OU

Karas, Vladimir, 1R, 4H

Karelin, D., IR

Karkour, N., 6C

Kaspi, Victoria, 17

Kataoka, Jun, OU, 11, 13

Kataria, Dhiren, 02, IR

Kathiravan, S., IF

Kato, Yuka, 3T

Katoch, Tilak, 1D

Katsuda, Satoru, OU

Katsukawa, Y., 3D

Katsuragawa, Miho, 5C

Katsuta, Junichiro, 0U, 11, 13

Kaufmann, P., $2 Q$

Kawabata, Tomoki, 57

Kawaharada, Madoka, ou

Kawai, Nobuyuki, OU

Kawano, Taro, OU, OX

Kawasaki, Shigeo, OU

Kazakevich, Y., 36

Kazeva, John D., 3Q

Kearney, John, 4X

Keek, L., $1 R$

Kelley, N. A., 2Q

Kelley, Richard L., $0 Q, 0 U, O W, O X, O Y, 1 N, 2 H, 3 L$,

$3 \mathrm{M}, 3 \mathrm{~N}, 3 \mathrm{O}, 3 \mathrm{Q}, 3 \mathrm{~S}, 3 \mathrm{~T}, 3 \mathrm{U}, 3 \mathrm{~W}$

Kellogg, James W., $1 \mathrm{H}$

Kelly, Daniel P., 3L

Kempf, Sascha, 3J

Kennedy, Patrick Kenneth, 5C

Kennedy, T., 1R, 2X

Kenyon, Steven J., 1H, 4X

Kerschbaum, Franz, 2D

Kester, T., IV

Khan, Aafaque R., 03

Khangulyan, Dmitry, OU

Kiener, J., 2N, 6C

Kikuchi, Naomichi, 0Z, 3U, 3X, 3Y, 3Z, 5D

Kilaru, K., $1 \mathrm{~J}$

Kilbourne, Caroline A., OQ, OU, OW, OX, OY, 2H, 2I,

$3 \mathrm{~L}, 3 \mathrm{M}, 3 \mathrm{~N}, 3 \mathrm{O}, 3 \mathrm{Q}, 3 \mathrm{~S}, 3 \mathrm{~T}, 3 \mathrm{U}, 3 \mathrm{~W}, 5 \mathrm{~S}$

Kim, Juyong, $1 \mathrm{~A}$

Kimball, Mark O., 0U, 30

King, Ashley, ou

Kink, Walter, $1 \mathrm{~K}$

Kintziger, Christian, 07, 2W

Kirichenko, Alexey S., 3B

Kishimoto, T., 2M

Kishimoto, Yuji, 1 A

Kissel, S., 4C

Kitaguchi, Takao, 0U, 13

Kitamoto, Shunji, OU, OY, 35

Kitayama, Tetsu, OU

Kiviranta, Mikko, 2I, 5R, 5S, $5 \mathrm{~T}$ 
Klochkov, D., 1R

Kluzniak, W., 1R

Knödlseder, J., 2N

Knudsen, Erik B., 5O, 76

Kobayashi, Ken, 08, 3D, 73

Koch, Anna, 2C, 2D

Koechlin, L., 3E

Koenecke, Richard G., 0Z, 1H, 4X

Koerding, E., $1 \mathrm{R}$

Kohmura, Takayoshi, OU, 10

Kokkotas, K., IR

Kokubun, Motohide, OU, OX, 11, 13

Kole, M., 2P

Kolodziejczak, Jeffrey, 17, 1 J, 1V, 1X

Komossa, S., $1 \mathrm{R}$

Komura, S., $2 M$

Kong, M. N., $2 P$

Kong, S. W., 2P

Korpela, Eric, 38

Korpela, Seppo, 1R, 4Y

Kosaka, Tatsuro, OU, 12, 41

Kotak, Sanket, IE

Kotaka, T., 2R

Koujelev, Alex, OU

Kouveliotou, C., IR

Kowalski, A. F., IR

Koyama, Katsuji, OU

Koyama, Shu, OU, OY, 3S, 3T, 3U

Koyande, J., $1 \mathrm{E}$

Kozon, Robert P., $1 \mathrm{H}$

Kraft, Ralph, OL

Kretschmar, Peter, OU

Kreykenbohm, Ingo, 1K, IR

Krimm, Hans, OU, 14

Kripps, Kari L., 3Q

Krivchenko, Aleksandr, 1J, 50, 51

Krivonos, R., $1 \mathrm{~J}$

Krivova, Natalie, 03

Kroth, Udo, 47

Krucker, Säm, OE, OF

Kruczek, Nicholas, OA, 2V

Krumrey, Michael, 1I, 27, 5K, 5N, 5P

Krzanowski, James E., 6L

Kubo, Hidetoshi, 1A, 2M

Kubo, Masahito, 08, 3D

Kubota, Aya, OU

Kudelin, M., $1 \mathrm{~J}$

Kuiper, L. M., IR

Kumar, Amit, $1 \mathrm{~F}$

Kumar, P., 4N

Kume, Kyo, OM

Kunieda, Hideyo, OU, 12, 41

Kunneriath, D., 1R

Kurashima, Sho, 0Z, 3U, 3X, 3Y, 3Z, 5D

Kurkela, A., IR

Kuromaru, G., 1N

Kurosawa, S., $2 \mathrm{M}$

Kurubi, Hiroyuki, 57

Kuvvetli, Irfan, 1R, 2D

Kuwabara, Masaki, 3G
Kuzin, Sergei V., 3B

Kuznetsova, Maria, 1 J, 51

Labanti, C., 1R, 2N, 6l, 6J

Lachaud, C., IR

Lacombe, K., OJ

Ladiya, T., 4N, 6G

Lafay, $\mathrm{X} ., 6 \mathrm{C}$

La Franca, F., $1 R$

Lai, D., IR

Lairson, Bruce, $3 Q$

Lam Trong, Thien, 2G, 2I

LaMarr, Beverly, 1H, 1I, 4W

Lamb, F. K., IR

Lambertson, Mike D., $1 \mathrm{H}$

Lamer, Georg, IK

Lancelot, J. Paul, $1 \mathrm{~F}$

Landgraf, B., 5P

Lapshov, Igor, 1 J, 1K, 51

Larruquert, Juan Ignacio, 08

Larson, Anne M., $1 \mathrm{H}$

Latronico, Luca, 4G

Laubert, P. P., IR

Laubis, Christian, 11, 47

Laurent, Philippe, 0U, 11, 13, 2N, 42, 63, 6C

Laurenza, M., 2K, 63

Lawrence, David J., 6B

Leary, James $C$., 6B

Lebrun, François, OU, 11, 13, 1R

Lechanoine-Leluc, C., $2 \mathrm{P}$

Le Coguie, A., OS

Le Duigou, J. M., 4L

Lee, Sang-Jun, $0 Q$

Lee, Shiu-Huang, OU

Leenaarts, Jorrit, 08

Lefmann, Kim, 76

Legay, E., 6C

Legere, Jason S., 2O, 6K, $6 \mathrm{~L}$

Lemaitre, G., 31

Le Mer, Isabelle, OF

Lentine, Steven, $1 \mathrm{H}$

Le Provost, H., IR

Leutenegger, Maurice A., OU, OW, OY, 3L, 3Q, 3T, $3 \mathrm{U}, 3 \mathrm{~W}$

Levantino, Matteo, 60

Le Ven, V., 6C

Levin, Vasily, $1 \mathrm{~J}, 50,51$

Levin, Zuni, 3J

Lewis, Jesse $\mathrm{H} ., 1 \mathrm{H}$

Li, Lei, 02

Li, LU, 2P

Li, Ran, 6D

Li, X., IR

Li, Yong, 6D

Liang, E., $1 R$

Liao, Yingyu, 7E

Ligori, S., 62

Lillis, Robert, 38

Lilly, Michael G., 1H

Limousin, Olivier, OF, 0J, 0O, OU, 11, 13, 1R, 2N, 42, $6 \mathrm{C}$ 


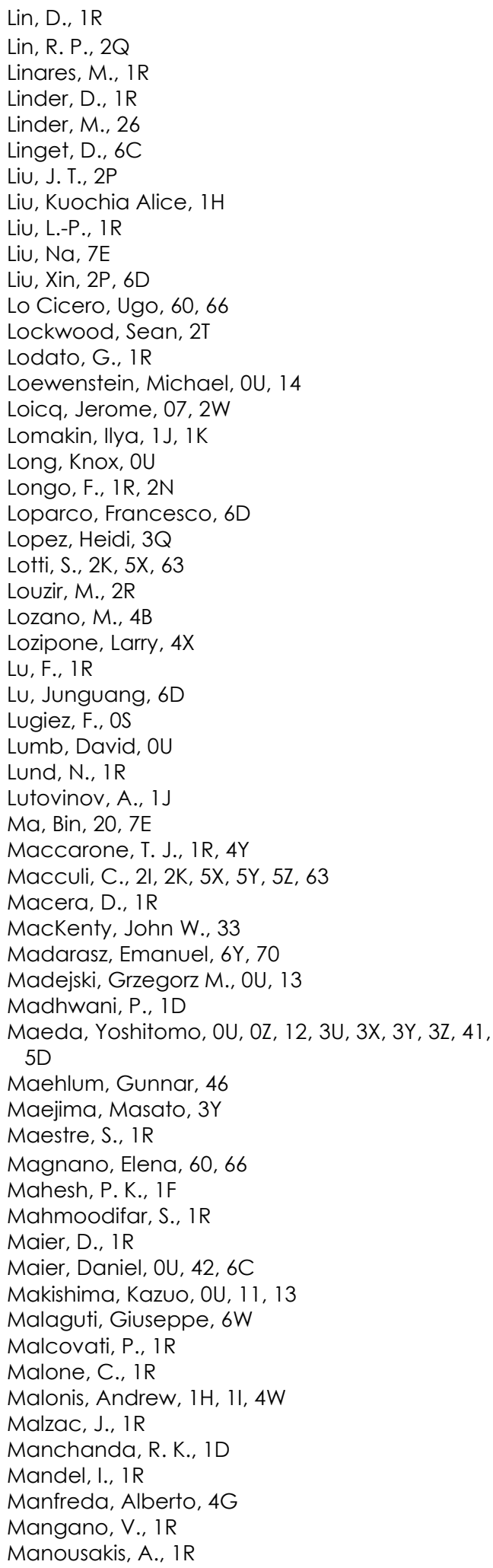

Manthripragada, Sridhar S., 1H

Marcinkowski, R., 2P

Marcos-Arenal, Pablo, 2Z, 30

Marelli, M., IR

Marggraf, Stefanie, 5K

Margueron, J., IR

Marinucci, A., 1R

Marisaldi, M., 1R, 2N, 6l, 6J

Markevitch, Maxim, OU, $3 \mathrm{U}$

Markoff, S. B., IR

Markowitz, A., IR

Markwardt, Craig B., 1H, 4W

Marquez, Vanessa, 73

Marsella, Giovanni, 6D

Marshall, Herman L., 17, 44, 56

Martignac, Jérôme, OF

Martindale, A., IR, IY

Martínez, G., 1 R

Martínez, R., 4B

Maruca, B. A., 2Q

Mason, James Paul, 09

Massa, F., 4l

Massahi, Sonny, 5K, 5O, 5P, 6P, 76

Massaro, E., 4

Masters, Candace, $0 \mathrm{U}, 3 \mathrm{~N}$

Masterson, R., 4C

Mates, Benjamin, $\mathrm{OQ}$

Mathew, Joice, 32, 39

Matonak, Bryan D., $1 \mathrm{H}$

Matsumoto, Hironori, OU, 12, 3Y, 3Z, 41, 71

Matsuoka, Y., 2M

Matsushita, Kyoko, OU

Matt, Giorgio, 17, 4H

Mazzarella, James R., 1S, 21, 6X

Mazziotta, Mario Nicola, 6D, 6E

Mazzoli, Alexandra, 07, 2X

McBreen, S., 2N

McBride, Stephen, OE

McCammon, Dan, OU, OW, OX, OY, 3L, 3M, 3N,

$3 Q, 3 U, 3 W$

McClelland, Ryan S., 1S, 6X, 7A

McConnell, Mark L., 2O, 6K, 6L

McCoy, Jake, 24

McCracken, Jeff, 73

McEnery, J. E., 2N

McEntaffer, Randall, 24, 4P

Mcginnis, Isaac E., $1 \mathrm{H}$

McGuinness, Daniel S., OU, 3M, 3Q

McHardy, I. M., IR

McKenzie, David E., 08

McNamara, Brian, OU

McPhate, Jason, OD, 38, 3F

Medina-Tanco, G., $1 R$

Mehdipour, Missagh, OU, IR

Meidinger, Norbert, 1K, 2A, 2B, 2C, 2D, 2E, 67, 68

Meier, Dirk, 46

Melatos, A., $1 \mathrm{R}$

Mende, Stephen B., 07, 2W

Menderov, A., $1 \mathrm{~J}$

Mendez, M., IR 
Mercier, K., OJ, 4L

Mereghetti, S., 1R, 2N

Merloni, Andrea, 1K, 2B

Meuris, Aline, OF, 0O, 4L

Mican, Benjamin, $1 \mathrm{~K}$

Michalska, Malgorzata, 1R, 4Y

Migliari, S., IR

Mignani, R., $1 R$

Mihara, T., IR

Mihara, Tatehiro, 1A

Miko, Joseph, OU

Miller, Eric D., OU, 14, 4P

Miller, Jon M., OU, 1R, 4P

Miller, M. C., IR

Miller, Richard S., 6B

Miller, Roger L., $1 \mathrm{H}$

Miller, Tim, 07, 38

Milliard, Bruno, 31, 31

Minamiyama, $Y ., 2 R$

Mineo, T., 1R, 2K, 52, 63

Mineshige, Shin, OU

Minesugi, Kenji, OU, OX

Miniussi, Antoine R., OQ, 2H

Miniutti, G., $1 R$

Minuti, Massimo, 4G

Mirabel, F., 2N

Mirc, Frédéri, 3l

Mirza, Irfan, $1 \mathrm{E}$

Misra, Ranjeev, 1D

Mitchell, Alissa L., 1H

Mitchell, Jason W., $1 \mathrm{H}$

Mitsuda, Kazuhisa, OU, OW, OX, OY, 1N, 2J, 3N, 30 3P, 3Q, 3R, 3S, 3T, 3U, 3W

Mitsuishi, Ikuyuki, OU, OY, 12, 1N, 3P, 3R, 3S, 3Z, 41, 71

Miuchi, K., 2M

Miyamoto, S., 2M, 2R

Miyaoka, Mikio, 35

Miyazawa, Takuya, OU, 0Z, 12, 3Y, 41

Mizumoto, T., $2 \mathrm{M}$

Mizumura, $Y ., 2 M$

Mizuno, Tsunefumi, 0U, 11, 13, 1A

Mizushima, Satoshi, OM

Mochizuki, B. A., 2Q

Mohammed, Jelila S., $1 \mathrm{H}$

Mohan, Rekhesh, $1 \mathrm{~F}$

Molendi, S., 2K, 63

Molkov, S., $1 \mathrm{~J}$

Mondal, Chayan, $1 \mathrm{~F}$

Monroe, Charles A., $1 \mathrm{H}$

Montel, Johan, 3I

Montt de Garcia, Kristina M., 1H

Moore, Christopher Samuel, 09

Morgan, Kelsey M., OQ, 2H

Mori, Hideyuki, OU, OZ, 12, 3U, 3X, 3Y, 3Z, 41, 5D

Mori, Koji, OU, 10

Mori, Kunishiro, 11, 13

Mori, Nicola, 6D

Moroso, Franco, OU

Morselli, A., 2N
Morsink, S., IR

Moseley, Harvey, OU

Moseley, Samuel J., 3M, 3Q

Motch, C., IR

Motta, S., IR

Mouchet, M., $1 R$

Mouret, G., IR

Muench, Theodore, 0U, 30

Mukai, Koji, OU

Mukerjee, Kallol, $1 \mathrm{E}$

Mulačová, J., IR

Mulé, Peter D., 1H

Muleri, Fabio, 17, 1R, 4G, 4H, 4I

Müller, Peter, $5 \mathrm{~N}$

Müller, Siegfried, $1 \mathrm{~K}$

Müller-Seidlitz, Johannes, 2C, 67

Muñoz-Darias, T., IR

Murakami, Go, 3G

Murakami, Hiroshi, OU, OY, 10

Murakami, Masahide, OY, 3P

Murakami, Toshio, OU

Murthy, Jayant, 1F, 32, 39

Mushotzky, Richard, OU, 4P

N.P. S., Mithun, 1G, 4D

Nagabhushana, S., IF

Nagano, Hosei, OU, 12, 41

Nagao, Louis T., $1 \mathrm{H}$

Nagino, Ryo, OU, 10

Najafiuchevler, Bahram, 46

Nakagawa, Takao, OU

Nakajima, Hiroshi, OU, 10, 57

Nakamasu, Y., $2 \mathrm{M}$

Nakamori, Takeshi, 0U, 11, 13, 1A

Nakamura, Yasuo, OX

Nakaniwa, Nozomi, 0Z, 3U, 3X, 3Y, 5D

Nakano, Toshio, 0U, 11, 13

Nakashima, Shinya, OU

Nakayama, Satoshi, 2U

Nakazawa, Kazuhiro, OU, 11, 13, 2N

Namba, Yoshiharu, OU, 12, 41

Nandra, Kirpal, 1K, 2A, 2B, 4H, 4P

Nandy, Dibyendu, 03

Narasaki, Katsuhiro, 0X, 3S

Narukage, Noriyuki, 08, 3D

Nasser, G., OJ

Natsukari, Chikara, OU, OX, 10

Navalkar, Vinita, 1E

Neguervela, I., IR

Neilsen, J., IR

Neiner, Coralie, $2 Y$

Nell, Nicholas, 0A, 2V

Neubert, T., IR

Ngo, Son N., $1 \mathrm{H}$

Nicolosi, P., 3H

Nicot, Jean-Marc, 31

Niemeyer, Jason, 6X

Nikzad, Shouleh, 34

Ninkov, Zoran, 05

Nirmal, K., 39

Nishioka, Yusuke, OU, 10

xxiv

Proc. of SPIE Vol. 9905 990501-24 
Nitta, Kiyofumi, 32

Nobukawa, Kumiko K., OU, 10

Nobukawa, Masayoshi, 0U, 10, 14

Noda, Hirofumu, OU, OY, 11, 13, 3N, 3R, 3S

Nomachi, Masaharu, OU

Noonan, John, 3J

Norris, Eric D., $1 \mathrm{H}$

Norton, A. J., IR

Norwood, Dwight A., $1 \mathrm{H}$

Novotka, Joseph, $1 \mathrm{H}$

Nowak, Michael A., 1R, 4P, 56

Nucita, A., $1 R$

Numata, Ai, 4X, 6X

Nuzzo, Flavio, 60

Obara, Shingo, $0 \mathrm{X}$

Oberlack, U., 2N

O'Brien, Paul T., 1M, 1R, 1Y, 5F

Oda, M., 2M

Odaka, Hirokazu, OU, 11, 13

O'Dell, Stephen L., OU, 17, 1 J, 1X

Oertel, M., IR

Ogasaka, Yasushi, 12, 41

Ogawa, Hiroyuki, OU

Ogawa, Mina, OU, OX, OY

Oger, R., 6C

Ogi, Keiii, OU, 12, 41, 71

Ohashi, Takaya, OU, 1N, 3P, 3R, 3S

Ohkuma, H., 2R

Ohno, Masanori, oU, 11, 13

Ohta, Masayuki, OU, 11, 13

Okajima, Takashi, OU, 0Z, 12, 1H, 3U, 3X, 3Y, 3Z,

$41,4 \mathrm{~V}, 4 \mathrm{X}, 5 \mathrm{D}$

Okamoto, Atsushi, OU, OX, 3R, 3S

Okazaki, Tsuyoshi, ou

Oleinikov, Vladimir, 51

Oliveira, Cristina, $2 T$

Olsen, Alf, 46

Olsen, Lawrence G., 0Z, 1H, 4X, 6X

Olsen, P. E. H., IR

Olson, J., 2Q

Onyeachu, Chimaobi O., $1 \mathrm{H}$

Orienti, M., $1 \mathrm{R}$

Origné, A., 31

Orio, M., $1 R$

Orlandini, M., IR

Orleanski, P., IR

Orosco, Henry Y., $1 \mathrm{H}$

Orsi, Silvio, 2P, 6D

Osborne, J. P., 1L, 1R, 1Y, 4L

Oscar, Adriani, 6D

Osten, R., IR

Ota, Naomi, OU, OY, IN

Otnes Berge, Hans Kristian, 46

Otsuka, Kiyomi, 0X, 3S

Ott, Sabine, 2C, 2D, 67

Ottensamer, Roland, 2D

Øya, Petter, 46

Ozaki, Masanobu, 0U, 10

Ozel, F., IR

Ozimek, Martin T., 6B
Pacciani, L., IR

Paerels, Frederik, OU, IR

Pahari, Mayukh, 1D

Påhlsson, Philip, 46

Palgan, T., 68

Paltani, Stéphane, OU, OY, 1R, 3U, 61

Panchuk, Vladimir E., 2Z, 35, 37

Panda, Dipak K., 6G

Paolillo, M., IR

Papadakis, I., IR

Papitto, A., IR

Pappas, C. G., 2H

Paragi, Z., IR

Paredes, J. M., IR

Pareschi, Giovanni, 23, 27, 50, 74

Parker, Joel Wm., 3J

Parmar, Arvind, OU

Parodi, G., 68

Pascal, S., 31

Patel, A. R., 4N, 6G

Pati, Ashok K., IF

Patinge, A., 6G

Patruno, A., IR

Paul, B., 1D, 1R

Pavlinsky, Mikhail, 1J, 1K, 50, 51

Pearce, Mark, 4H, 6D, 6E

Pearson, J. F., 1Y, 4L

Pederiva, F., $1 R$

Peille, Philippe, 2H, 5F, 5T, 5W, 64

Pellegrini, G., 4B

Pellizzoni, A., IR

Penacchioni, A. V., 1R

Penton, Steven, 2T

Peplowski, Patrick N., 6B

Perea Abarca, Belén, 2Z, 30

Peretz, U., IR

Perez, M. A., $1 R$

Perez-Torres, M., 1R

Perinati, E., 1R, 52, 65

Pérot, Etienne, 3l

Pertenais, Martin, $2 Y$

Pesce-Rollins, Melissa, 4G

Peterson, B. M., lR

Peterson, Jacqualine R., $1 \mathrm{H}$

Petit, Pascal, 2 Y

Petit, S., IY

Petracek, V., IR

Petre, Robert, OU, 3U, 4P

Pevear, Kristina N., $1 \mathrm{H}$

Peyré, J., 2N, 6C

Pfeffermann, Elmar, 1K

Pham, Karen K., $1 \mathrm{H}$

Piano, G., $2 \mathrm{~N}$

Picciotto, A., 61

Piemonte, C., 6l, 6J

Pietschner, Daniel, 1K, 2A, 2E

Pigot, C., OS

Pilch, A., 68

Pinchera, Michele, 4G, 4H

Pinsard, F., 4L 
Pinto, Ciro, OU

Piro, Luigi, 2I, 2K, 5F, 5W, 5X, 5Y, 5Z, 63

Píš, Igor, 60, 66

Pittori, C., 1R, $2 \mathrm{~N}$

Plastinin, Jurii A., 3B

Plattner, Markus, 2A, 2D, 68

Plesha, Rachel, 2T

Plucinsky, Paul P., 44, 45

Pohl, Martin, OU, 1R, 2P, 4Y, 6D, 6E

Poilleux, P., 2R

Pointecouteau, Etienne, 5W

Polak, S., 68

Pollard, Sue E., $1 \mathrm{H}$

Pons, J., IR

Pons, R., OJ

Pontius, James T., OU, OX

Pope, John S., $1 \mathrm{H}$

Poppenhaeger, K., 4P

Poppett, Claire, 2W

Porro, Matteo, 2C

Portell, J., IR

Porter, Frederick S., OQ, OU, OW, OX, OY, 2H, 3L,

$3 \mathrm{M}, 3 \mathrm{~N}, 3 \mathrm{O}, 3 \mathrm{Q}, 3 \mathrm{~S}, 3 \mathrm{~T}, 3 \mathrm{U}, 3 \mathrm{~W}$

Possenti, A., IR

Postma, Joe, $1 \mathrm{~F}$

Postnov, K., IR

Pottschmidt, Katja, OU

Poutanen, Juri, 1R, 4H

Powers, Charles E., $1 \mathrm{H}$

Powers, Daniel F., $1 \mathrm{H}$

Prakash, Ajin, 32, 39

Prakash, M., 1R

Prandoni, I., IR

Pratt, Gabriel W., 2B

Predehl, Peter, $1 \mathrm{~K}$

Prêle, D., 5 U

Price, Samuel R., $1 \mathrm{H}$

Prigozhin, Gregory Y., 1H, 1I, 4V, 4W

Probst, Anne-Catherine, $6 \mathrm{~V}$

Produit, N., 2P

Proffitt, Charles, 2T

Proserpio, Laura, 6V, 6Y, 70

Psaltis, D., 1R

Ptak, A., 4P

Puigdengoles, C., 4B

Purves, Lloyd, 05

Pye, J., IR

Qi, Runze, 6Q

QU, J., 1R

Quan, Zheng, 6D

Quijada, Manuel A., OA

Quiret, S., 31

Raab, Walfried, 02

Rachevski, A., 1R, 6I, 6J

Rachmeler, Laurel A., 08

Raffanti, Rick, 3F

Rajarshi, Chaitanya, 03

Rambaud, D., OJ, IR

Ramirez, Julian B., $1 \mathrm{H}$

Ramon, Pascale, OJ, $1 \mathrm{R}$
Ramaprakash, A. N., 03

Ramsay, G., IR

Ramsey, Brian D., 0E, OU, 17, 1J, 1V, 73

Rao, A. R., 1G, 4D

Rao, N. Kameswara, $1 F$

Rapin, D., 2P

Rapisarda, M., IR

Rashevskaya, I., 1R, 6I, 6J

Rataj, M., 68

Rau, Arne, 2A, 2B, $5 \mathrm{G}$

Rauly, E., 6C

Rauw, G., 61

Ravensberg, Kevin, $5 \mathrm{~S}$

Ray, Paul S., IR, 4Y

Rea, N., IR

Read, Andy, 02

Rebuffat, Denis, 02

Reddy, S., IR

Reglero, Victor, $4 \mathrm{H}$

Reichard, Karl, OL

Reichel, Thomas, 47

Reid, Winston J., $1 \mathrm{H}$

Reiffers, Jonas, 2D

Reig, P., IR

Reina Aranda, M., $1 R$

Reintsema, Carl D., OQ, 2H

Reiprich, Thomas H., 2B

Remillard, Ronald A., 1H, 1I, 1R, 4W

Renaud, Diana, 42

Renotte, Etienne, 2W, 2X

Reva, Anton A., 3B

Revnivtsev, M., $1 \mathrm{~J}$

Reynolds, Christopher, OU, IR

Rezzolla, L., IR

Ribo, M., $1 \mathrm{R}$

Richter, Rainer H., 67

Rider, Kodi, 2 W

Riggio, A., $1 R$

Rignanese, L. P., 6I, 6J

Rios, A., IR

Rischke, D. H., $1 R$

Riveros, Raul E., 1S, 21

Rizvanov, Artem A., 3B

Robberto, Massimo, 05

Robinson, David, $\mathrm{OZ}$

Robrade, Jan, $1 \mathrm{~K}$

Roche, J., IV

Rochus, P., $2 X$

Rodriguez, J., $1 R$

Rodriguez, M., OS

Rodríguez-Gil, P., 1R

Rogstad, Eric M., $1 \mathrm{H}$

Rohlfs, R., IR

Roman-Duval, Julia, 2T

Romani, Roger, 17

Romano, P., IR

Romstedt, Jens, 02

Rosecrans, Glenn P., $1 \mathrm{H}$

Rosier, P., 6C

Rossi, C., 5 Y 
Rossi, E. M. R., IR

Rotin, Alexey, 1 J, 50, 51

Rousseau, A., $1 R$

Roux, W., 3E

Rowe, John N., $1 \mathrm{H}$

Różańska, Agatha, 1R, 2B

Rubini, Alda, 4G, 4H

Rudak, B., $1 R$

Runciman, Chris, 02

Russell, D. M., IR

Russell, Helen, OU

Rutczynska, A., 2P

Rutkowski, Kristin, 14

Ryan, James M., 2O, 6K

Rybka, D., 2P

Ryde, Felix, 1R, 6D

Ryu, K., 4C

Sabatini, S., $2 \mathrm{~N}$

Sabau, Maria Dolores, $4 \mathrm{H}$

Sabau-Graziati, L., $1 \mathrm{R}$

Sachkov, Mikhail, 04, 2Z, 30, 35, 36, 37

Sadamoto, Masaaki, $1 \mathrm{~A}$

Sadleir, John E., OQ, 2H

Safi-Harb, Samar, OU

Safonova, Margarita, 32, 39

Sager, Jennifer A., $1 \mathrm{H}$

Saha, Timo T., 1S, 6X, 7A

Sahin, H., OS

Sahnow, David J., 2T

Sahu, Snehalata, IF

Saint-Hilaire, P., 2Q

Saito, Shinya, OU, 11, 13

Saji, Shigetaka, $3 z$

Sakai, Kazuhiro, $0 Q, 0 U, 1 N, 2 H, 2 Q$

Sakai, Shin-ichiro, OU

Sakamoto, T., IR

Sala, G., IR

Salmaso, B., 23, 50

Salvaterra, R., IR

Salvato, Mara, $1 \mathrm{~K}$

Salvetti, D., IR

Sameshima, Hiroaki, OU

Sample, J., $2 Q$

Sandberg, J., $1 R$

Sanders, Claude A., $1 \mathrm{H}$

Sanders, Jeremy S., 2B, 4P

Sankarasubramanian, Kasiviswanathan, IF

Sanna, A., IR

Santangelo, Andrea, 1K, 1R, 4H, 4Y, 52, 65

Santos, C., 6C

Sarbadhikari, A. Basu, 6G

Sargent, Andrew, 14

Sarpotdar, Mayuresh, 32, 39

Sasaki, Manami, 1K, 2B

Sasaki, Toru, OU

Sato, Goro, OU, 11, 13, 5C

Sato, Kosuke, OU, OW, OY, 3S, 3U, 3W

Sato, Rie, 0U, 11, 13

Sato, Toshiki, OZ, 3U, 3X, 3Y, 3Z, 5D

Sato, Yoichi, OU, OX, OY, 3S
Sauvageot, J. L., OS

Savadkin, Bruce, $1 \mathrm{H}$

Savage, Sabrina, 73

Savolainen, T., 1R

Sawada, Makoto, OU, OW, OX, OY, 14, 3S, 3T, 3U, 3W

Sawano, Tatsuya, 0M, 2M

Saylor, Maxine R., $1 \mathrm{H}$

Sazonov, S., 1J

Scaringi, S., IR

Schaeffer, Alexander F., 1H

Schaffner-Bielich, J., IR

Schanne, Stephane, 1R, 4Y

Schanz, Thomas, 2D

Schartel, Norbert, OU

Schattenburg, Mark L., 1X, 22, 4P, 73

Schatz, H., IR

Schee, J., IR

Scheverle, Hartmut, IK

Schiminovich, David, 31, 31

Schindhelm, Eric, 3J

Schmid, C., IR

Schmitt, Jürgen, $1 \mathrm{~K}$

Schmutz, W., 2X

Schneider, R., IR

Schnell, Andrew, 4Y

Schnittman, J. D., IR

Schofield, Mark J., 6X

Scholze, Frank, 11, 47

Schouten, R. M., 5V

Schreiber, S., 5P

Schühle, U., $2 X$

Schulz, Norbert S., 4P, 56

Schweiss, Andrea N., 3Q

Schweiss, Nancy S., $1 \mathrm{H}$

Schwenk, A., IR

Schwope, Axel, 1K, IR

Schyns, E., IY

Sciortino, Luisa, 60, 66

Sciortino, S., 61

Scott, Christopher J., 6B

Sedrakian, A., IR

Seljak, Andrej, 3F

Sembay, Steve, 02

Semena, N., 1 J, 50

Semeniouk, I., 2R

Semper, Sean R., 1H

Serbinov, D., $1 \mathrm{~J}, 50$

Serino, M., IR

Serlemitsos, Peter J., OU, 0Z, 1H, 3U, 3X, 5D

Seta, Hiromi, OU, OW, OY, 3S, 3T, 3U, 3W

Seyler, J.-Y., IR

Sgrò, Carmelo, 4G, 4H

Shackelford, Larry V., $1 \mathrm{H}$

Shah, Harshit, 1E

Shah, Parag, 1D

Shakura, N., IR

Shanmugam, M., $4 \mathrm{~N}$

Sharma, Tejaswita, 6K

Sharpe, Marton V., 21, 6X 
Shearer, A., IR

Shen, Zhengxiang, 20

Shi, Dalian, 6D

Shi, H. L., 2P

Shibano, Yasuko, OU

Shida, Maki, OU

Shidatsu, Megumi, OU

Shih, A. Y., 2Q

Shimada, Takanobu, OU

Shimizu, Toshifumi, $2 U$

Shinozaki, Keisuke, OU, OY, 2J, 3S

Shirazi, Farzane, 6L

Shirron, Peter J., OU, OY, 3N, 3O, 3P, 3R, 3S

Shkolnik, Evgenya, 34

Shore, S., IR

Shortt, Brian, 27, 5K, 5O, 5P, 76

Shourt, William Van, OE, 38

Shukla, P., 4N

Shuman, S., 3M

Shustov, Boris, 04

Sichevskij, S., 36

Siegmund, Oswald H. W., OA, OD, 38, 3F

Simionescu, Aurora, OU

Simmons, Cynthia, OU

Sims, M., $1 R$

Singh, Kulinder Pal, $1 \mathrm{E}$

Sizun, $P$., $2 R$

Skup, Konrad, 2D

Slowikowska, A., 1R

Smale, A., 4P

Smith, A., IR

Smith, D. M., 1R, 2Q

Smith, P. J., 1R, 2X

Smith, Randall K., OU, 4P

Smith, Stephen J., 0Q, 2H, 2I, 5S, 5T, 5W, 64

Smith, W. S., IV

Sneiderman, Gary A., OU, OW, OX, OY, 3N, 3O, 3S, 3U, 3W

Sobolewska, M., IR

Sochora, V., IR

Soffitta, Paolo, 17, 1R, 4G, 4H, 4I

Solanki, Sami K., 03

Soleri, P., IR

Solly, Peter M., 1S, 7A

Solstad, Mathias, 76

Song, L. M., 1R, 2P

Sonnentrucker, Paule, 2T

Sonoda, S., 2M

Soong, Yang, OU, 0Z, 1H, 3U, 3X, 4X, 5D

Sordet, M., 61

Soufflet, Fabrice, OF

Spada, Francesca, 4G, 4H

Spandre, Gloria, 4G

Spanswick, Emma, 02

Spencer, A., 1R

Spiga, Daniele, 23, 27, 50, 6R, 6W, 76

Spillantini, Piero, 6D

Sreedhar, Yuvraj Harsha, IF

Sreejith, A. G., 32, 39

Sreekumar, P., $1 F$
Sreekumar, S., $1 \mathrm{G}$

Srinivas, A. R., 4N

Sriram, S., 03, $1 \mathrm{~F}$

Stalin, Chelliah S., IF

Stamerra, A., IR

Stappers, B., $1 R$

Staubert, R., IR

Stawarz, Łukasz, OU, 2N

Steenari, David, 46

Stefanescu, A., 26

Steffl, Andrew, 3J

Stein, Timo A., 46

Steiner, A. W., $1 R$

Stella, Luigi, 1R, $4 Y$

Steller, Manfred, 2D

Štěpán, Jiri, 08

Stergioulas, N., IR

Stern, S. Alan, 3J

Stevens, A. L., IR

Stevenson, Thomas R., OQ

Stewart, Gordon C., $1 \mathrm{E}$

Stockman, Yvan, 2W

Stollenwerk, Manfred, 6V

Stratta, G., IR

Strecker, Rafael, 2A

Strohmayer, T. E., IR

Struebel, Jonathan, $1 \mathrm{H}$

Stuchlik, Z., IR

Stutz, Stefan, OF

Su, Meng, 6D, 6E

Subramaniam, Annapurni, $1 \mathrm{~F}$

Subramanian, K. P., 4N

Suchy, S., IR

Suematsu, Yoshinori, 08, 3D

Sugawara, Yasuharu, OU

Sugita, Hiroyuki, OU, OX, OY, 35

Sugita, Satoshi, OU, 0Z, 12, 3U, 41, 71

Suleimanov, $V_{\text {., }} 1 \mathrm{R}$

Sun, J. C., $2 P$

Sun, Xin, 6D

Suntharalingam, V., 4C

Sunyaev, Rashid, 1J, 1K

Surdo, Antonio, 6D

Sutaria, Firoza, IF

Suzuki, Fumiharu, $3 G$

Suzuki, S., $1 \mathrm{~N}$

Suzuki, Yoshio, 12, 41

Swanson, Jack, 2V

Swartz, D., 1J

Swetz, Daniel S, OQ, $2 \mathrm{H}$

Sykes, Jon M., 02, 4L

Szabelski, J., 2P

Szymkowiak, Andrew E., OU, OW, OY, 3L, 3N, 3U, $3 \mathrm{~W}$

Tachibana, Kenji, $\mathrm{OZ}$

Tachibana, Sasagu, $3 Z$

Tagliaferri, Giampiero, 4H

Tajima, Hiroyasu, OU, 11, 13

Tajima, Takao, $2 \mathrm{U}$

Takada, A., 2M 
Takahashi, Hiromitsu, OU, 11, 13, 14, 1A

Takahashi, Tadayuki, OE, OU, OX, 11, 13, 1R, 5C

Takeda, Sawako, 3T

Takeda, Shin'ichiro, OE, OU, 11, 13, 5C

Takei, Yoh, OU, OW, OX, OY, 1N, 3N, 3O, 3Q, 3R, 3S, 3U, 3W

Takemoto, A., $2 \mathrm{R}$

Takemura, T., $2 \mathrm{M}$

Talebi, Jahanzad, 46

Tamagawa, Toru, OU, OY, 3S

Tambov, V., 50

Tamburini, F., IR

Tamura, H., IR

Tamura, Keisuke, OU, 0Z, 12, 3Y, 41

Tamura, Takayuki, oU

Tanaka, Takaaki, OU, 10, 13

Tanaka, Yasuo, OU

Tanaka, Yasuyuki, 0U, 13

Tandon, Shyam N., IF

Tanimori, T., $2 \mathrm{M}$

Tapie, Pierre, 31

Tashiro, Makoto S., OU, OW, OY, 3S, 3T, 3U, 3W

Tatischeff, $\mathrm{V}_{\text {., }} \mathrm{N}$, 6C

Tauris, T., IR

Tavani, M., 2N

Tavecchio, F., $1 \mathrm{R}$

Tawara, Yuzuru, OU, 0Z, 12, 1N, 3U, 41, 71

Tayabaly, K., 50

Taylor, Joanna, 2T

Tedesco, J., OD

Temi, P., 4P

Tennant, Allyn, 17

Tenzer, Christopher, 1K, 1R, 2D, 4H, 52, 65

Terada, Yukitatsu, OU, OY, 1 1, 13, 14, 3T

Terashima, Yuichi, OU

Theobald, Craig, 4H

Theodorou, Theodorus, $4 \mathrm{H}$

Thielemann, F. K., IR

Thomas, Chris, 2D

Thompson, D. J., 2N

Thornhill, Julian, 02

Thorpe, Rosemary S., 3Q

Tiengo, Andrea, 1R, 6W

Tiwari, Neeraj K., 4N

Tkachenko, A., $1 \mathrm{~J}$

Tolos, L., IR

Tombesi, Francesco, OU, IR

Tomida, Hiroshi, OU, 10

Tomono, D., 2M

Tomsick, John A., 1R, 6P

Torok, G., IR

Torras-Rosell, Antoni, 5K

Torrejon, J. M., $1 R$

Torrentó, A.-S., 6C

Torres, D. F., IR

Torresi, E., IR

Torrioli, G., 2K, 5X

Tourrette, Thierry, OF, 4L

Tramacere, A., IR

Traub, Wesley, 34
Traulsen, I., IR

Treberspurg, Wolfgang, 2C, 67

Treis, Johannes, 67

Tremsin, Anton, 3F

Tripathi, Durgesh, 03

Trois, A., IR

Trujillo-Bueno, Javier, 08, 3D

Tsavalas, John G., 6L

Tsuboi, Yohko, OU, 5E

Tsujimoto, Masahiro, OU, OW, OY, 3N, 3S, 3T, 3U, $3 \mathrm{~W}$

Tsunematsu, Shoji, 0X, 3P, 3S

Tsunemi, Hiroshi, OU, 10, 57

Tsuru, Takeshi Go, OU, 10

Turin, Paul, OE

Turolla, R., $1 \mathrm{R}$

Turriziani, S., IR

Typel, S., $1 R$

Uchida, Hiroyuki, OU, 10

Uchiyama, Hideki, OU, 11, 13

Uchiyama, Yasunobu, 0U, 11, 13

Ueda, Shutaro, OU, 10

Ueda, Yoshihiro, OU

Ueno, Shiro, OU

Uesugi, Kentaro, 12, 41

Ullán, M., 4B

Ullom, Joel N., OQ, 2H

Ulyanov, A., $2 \mathrm{~N}$

Uno, Shin'ichiro, OU

Urry, Meg, OU

Ursino, Eugenio, ou

Uruga, Tomoya, $3 Z$

Uslenghi, M., 2K, 61

Uter, P., IR

Uttley, P., IR

$\checkmark$. Kienlin, Andreas, $1 \mathrm{~K}$

Vacanti, Giuseppe, 27, 5N

Vacchi, A., 1R, 6l, 6J

Vadawale, Santosh V., 1G, 4D, 4N, 6G

Valencic, L., 4P

Valenziano, L., 62

Vallerga, John, OD, 3F

Valsecchi, Giuseppe, 27

van Baren, Coen, 1R, 27

van der Klis, Michiel, IR, 4Y

van der Kuur, Jan, 2H, 2I, 5R, 5S, 5T, 5V

van der Linden, A. J., 5S

van Leeuwen, B.-J., 2l, 5R

van Weers, Henk, 2I, 2J

Varner, Gary, 3F

Varniere, P., IR

Vasisht, Gautam, 34

Vassal, Marie-Cécile, OF

Vaughan, Simon, 1R, 2B

Vecchi, G., 23, 78

Verbeeck, C., $2 X$

Vercellone, S., $1 \mathrm{R}$

Vernani, Dervis, 27

Vezie, Michael L., 1H, 11

Vibhute, Ajay M., $1 \mathrm{G}$ 
Vietri, M., IR

Vievering, Juliana, OE

Vikhlinin, Alexey, $0 Q$

Villasenor, Joel S., 1H, $1 \mathrm{I}$

Vincent, F. H., IR

Vink, Jacco, 4H

Virta, Vihtori, 3F

Vishnyakov, Eugene A., 3B

Vishwakarma, S., $1 \mathrm{E}$

Voisin, F., $5 \mathrm{U}$

Vola, Pascal, 31

Von Ballmoos, Peter, 2N, 6P

Voronkov, S., $1 \mathrm{~J}$

Vrba, V., IR

Wada, Atsushi, OU, OX

Wade, Colin, 6P

Waegebaert, V., 0J

Wakeham, Nicholas A., OQ, 2H

Walk, S. J., 1 R

Walter, Roland, 2N, 6D, 6E

Walton, D., 1R

Wampler-Doty, Matthew P., $4 \mathrm{~V}$

Wang, Bo, 6D

Wang, Chi, 02

Wang, Haifeng, 20

Wang, Junjing, 1R, 6D

Wang, Kun, 20

Wang, Le, 6D

Wang, Ruijie, 2P, 6D

Wang, S., 2R

Wang, Xiaogiang, 20

Wang, Z., IR

Wang, Zhanshan, 20, 6Q, 7E

Wang, Zhigang, 6D

Wanlin, E., 6C

Wassell, Edward J., OQ, $2 \mathrm{H}$

Watanabe, Shin, OE, OU, 11, 13, 1R, 5C

Watanabe, Tomomi, OU, OW, 3L, 3M, 3Q, 3T, 3U, $3 \mathrm{~W}$

Watts, Anna, 1R, 4Y

Wawrzaszek, R., IR

Webb, N., $1 \mathrm{R}$

Webster, Chris, 34

Wei, Zhenbo, 7E

Weinberg, N., 1R

Weisskopf, Martin C., 17, 4H

Wen, Mingwu, 6V, 6Y

Wen, $X$., 2P

Wende, H., $1 R$

Werner, Norbert, OU

Westergaard, Niels J., 50, 76

Wheatley, P., IR

White, James, 2T

Wielders, Arno, 02

Wijers, R., IR

Wijnands, R., $1 R$

Wik, Daniel, OU

Wild, Christopher, OF

Wilke, Paul, OX

Wilkins, Dan, OU
Wille, Eric, 26, 27, 29, 5N, 50

Wille, M., 1R

Williams, Brian, OU

Willingale, R., 1L, 1Y, 4L, 4P

Wilms, Jörn, 1K, 1R, 2B, 4P, 5F, 5G, 5T, 5W, 64

Wilson, Jacob, $2 \mathrm{~V}$

Wilson-Hodge, Colleen A., 1R, 4Y

Winebarger, Amy R., 08, 3D, 73

Winter, Berend, IR, 4H, 4Y

Winternitz, Luke B., $1 \mathrm{H}$

Wishnow, Edward, 38

Witthoeft, Michael, 14

Wofford, George I., $1 \mathrm{H}$

Wolk, S., 4P

Wong, Emily N., $6 \mathrm{~L}$

Wood, K., IR

Woods, Thomas N., 09

Woosley, S. E., IR

Wright, Alex M., 6K

Wright, Graham, 22

Wright, Michael R., $1 \mathrm{H}$

Wu, Bobing, 2P, 6D

WU, X., IR

Wu, Xin, 2N, 6D, 6E

Xiao, H. L., 2P

Xiong, S. L., 2P

$X U, H . H ., 2 P$

$X U$, Ming, 2P, 6D

$X U, R ., 1 R$

Yadav, J. S., ID

Yamada, Shinya, OU, OW, OX, OY, 1N, 3S, 3T, 3U, $3 \mathrm{~W}$

Yamada, Takahiro, oU

Yamaguchi, Hiroya, OU, 14

Yamaguchi, M., 2R

Yamamoto, R., $1 \mathrm{~N}$

Yamaoka, Kazutaka, 0U, 1 1, 13

Yamasaki, Noriko Y., OU, OX, OY, 1N, 2J, 3Q, 3R, 3S, 3U, 3W

Yamauchi, Makoto, $0 U, 10$

Yamauchi, Shigeo, 0U, 12, 41

Yan, Peng, 6D

Yang, Mike $\mathrm{Y}_{\text {., }} 1 \mathrm{H}$

Yang, Yang, 6Q

Yanson, Alexei, 27

Yao, Youwei, 22

Yaqoob, Tahir, OU, 14

Yaroshenko, Valeri, $1 \mathrm{~K}$

Yaskovich, A., $1 \mathrm{~J}$

Yasuda, Susumu, OX

Yatsu, Yoichi, OU, OY, 11, 13, 3S

Yonetoku, Daisuke, OM, OU, 13, 1A

Yoneyama, Tomokage, $1 \mathrm{~A}$

Yoon, Wonsik, OQ, 2H

Yoshida, Atsumasa, OU

Yoshida, Kazuki, OM

Yoshida, Masaki, 08

Yoshida, Seiji, OX, 3N, 3O, 3P, 3Q, 3R, 3S

Yoshikawa, Ichiro, 3G

Yoshikawa, K., 2M 
Yoshikawa, Shun, 3Y

Yoshinaga, Keigo, $1 \mathrm{~A}$

Yoshioka, Kazuo, $3 G$

Yoshioka, Kenya, 71

Young, C. Alex, 6B

YU, W., IR

Yu, Wayne H., $1 \mathrm{H}$

Yuan, F., IR

Yuan, W., IR

Yuan, Y., $1 R$

Yuasa, Takayuki, OU, 11, 13

Yushkin, Maksim, 2Z, 35, 37

Zampa, G., 1R, 6l, 6J

Zampa, N., 1R, 6l, 6J

Zampieri, L., IR

Zane, Silvia, 1R, 4H, 4Y

Zanetti, Davide, $4 \mathrm{H}$

Zavlin, V., $1 \mathrm{~J}$

Zdunik, L., IR

Zdziarski, A., 1R, 2N, 6E

Zech, A., IR

Zenone, Isabelle, 3 I

Zhang, Aibing, 02

Zhang, B., IR

Zhang, C., $1 R$

Zhang, L. Y., 2P

Zhang, Li, 2P, 6D

Zhang, S., IR

Zhang, Shuangnan, 1R, 2P, 6D

Zhang, William W., 1S, 21, 6X, 7A

Zhang, X. F., 2P

Zhang, Y. J., $2 P$

Zhang, Zhong, 20, 6Q

Zhao, Jun, 3L

Zheng, Jianhua, 02

Zhuravleva, Irina, OU

Zingale, M., IR

Zoghbi, Abderahmen, OU

Zoglaver, A., 2N, 2Q

Zorzi, N., 6l, 6J

Zuknik, Karl-Heinz, 27

Zuo, Heng, 22

Zuppella, P., $3 \mathrm{H}$

Zwart, F., IR

Zwolinska, A., 2P 
Proc. of SPIE Vol. $9905990501-32$

Downloaded From: https://www.spiedigitallibrary.org/conference-proceedings-of-spie on 26 Apr 2023 Terms of Use: https://www.spiedigitallibrary.org/terms-of-use 


\section{Conference Committee}

Symposium Chairs

Colin Cunningham, UK Astronomy Technology Centre

(United Kingdom)

Masanori lye, National Astronomical Observatory of Japan (Japan)

Symposium Co-chairs

Allison A. Barto, Ball Aerospace \& Technologies Corporation (United States)

Suzanne K. Ramsay, European Southern Observatory (Germany)

\section{Conference Chairs}

Jan-Willem A. den Herder, SRON Netherlands Institute for Space Research (Netherlands)

Tadayuki Takahashi, Japan Aerospace Exploration Agency (Japan)

Marshall Bautz, Massachusetts Institute of Technology (United States)

Conference Program Committee

Hisamitsu Awaki, Ehime University (Japan)

Didier Barret, Institut de Recherche en Astrophysique et Planétologie (France)

Marcos Bavdaz, European Space Research and Technology Centre (Netherlands)

Angela Bazzano, INAF - Istituto di Astrofisica e Planetologia Spaziale (Italy)

Steven E. Boggs, University of California, Berkeley (United States) João Braga, Instituto Nacional de Pesquisas Espaciais (Brazil) Jin Chang, Purple Mountain Observatory (China)

Marco Feroci, INAF - Istituto di Fisica dello Spazio Interplanetario (Italy)

Luigi Gallo, Saint Mary's University (Canada)

Neil A. Gehrels, NASA Goddard Space Flight Center (United States) James C. Green, University of Colorado at Boulder (United States)

Fiona Harrison, California Institute of Technology (United States)

Margarita Hernanz, Consejo Superior de Investigaciones Científicas (Spain)

Caroline A. Kilbourne, NASA Goddard Space Flight Center

(United States)

Olivier Limousin, CEA-Centre de SACLAY (France)

Hironori Matsumoto, Nagoya University (Japan) 
Mark L. McConnell, The University of New Hampshire (United States)

Kazuhiro Nakazawa, The University of Tokyo (Japan)

Kirpal Nandra, Max-Planck-Institut für extraterrestrische Physik

(Germany)

Shouleh Nikzad, Jet Propulsion Laboratory (United States)

Takaya Ohashi, Tokyo Metropolitan University (Japan)

Giovanni Pareschi, INAF - Osservatorio Astronomico di Brera (Italy)

Biswajit Paul, Raman Research Institute (India)

Mikhail N. Pavlinsky, Space Research Institute (Russian Federation)

Paul S. Ray, U.S. Naval Research Laboratory (United States)

Vincent Tatischeff, Institut National de Physique Nucléaire et de Physique des Particules (France)

Hiroshi Tsunemi, Osaka University (Japan)

Martin C. Weisskopf, NASA Marshall Space Flight Center (United States)

Richard Willingale, University of Leicester (United Kingdom)

Jörn Wilms, Friedrich-Alexander-Universität Erlangen-Nürnberg (Germany)

Shuangnan Zhang, Institute of High Energy Physics (China)

\section{Session Chairs}

1 Solar/UV I

Shouleh Nikzad, Jet Propulsion Laboratory (United States)

2 Solar/UV II

Marshall Bautz, Massachusetts Institute of Technology (United States)

3 Solar/UV III

Shuangnan Zhang, Institute of High Energy Physics (China)

4 Detector

Caroline A. Kilbourne, NASA Goddard Space Flight Center (United States)

$5 \quad$ NASA Mission Studies: Joint Session with Conferences 9904 and 9905 Mario R. Perez, NASA Headquarters (United States)

6 Detector II

Mikhail N. Pavlinsky, Space Research Institute (Russian Federation)

7 ASTRO-H I

Marshall Bautz, Massachusetts Institute of Technology (United States)

8 ASTRO-H II

Takaya Ohashi, Tokyo Metropolitan University (Japan) 
9 Polarization

Margarita Hernanz, Consejo Superior de Investigaciones Científicas (Spain)

10 ASTROSAT

Marshall Bautz, Massachusetts Institute of Technology (United States)

11 NICER

Marco Feroci, INAF - Istituto di Fisica dello Spazio Interplanetario (Italy)

12 SRG

Marco Feroci, INAF - Istituto di Fisica dello Spazio Interplanetario (Italy)

13 New Missions

Kazuhiro Nakazawa, The University of Tokyo (Japan)

14 Optics I

Marcos Bavdaz, European Space Research and Technology Centre (Netherlands)

15 Optics II

Giovanni Pareschi, INAF - Osservatorio Astronomico di Brera (Italy)

16 ATHENA I

Richard Willingale, University of Leicester (United Kingdom)

17 ATHENA II

Marcos Bavdaz, European Space Research and Technology Centre (Netherlands)

18 ATHENA III

Kirpal Nandra, Max-Planck-Institut für extraterrestrische Physik (Germany)

19 Gamma

Margarita Hernanz, Consejo Superior de Investigaciones Científicas (Spain) 
Proc. of SPIE Vol. 9905 990501-36

Downloaded From: https://www.spiedigitallibrary.org/conference-proceedings-of-spie on 26 Apr 2023 Terms of Use: https://www.spiedigitallibrary.org/terms-of-use 